\title{
ON EXTREME POINTS OF P-BOXES AND BELIEF FUNCTIONS
}

\author{
IGNACIO MONTES ${ }^{1}$ AND SEBASTIEN DESTERCKE ${ }^{2}$
}

\begin{abstract}
Aвstract. Within imprecise probability theory, the extreme points of convex probability sets have an important practical role (to perform inference on graphical models, to compute expectation bounds, ...). This is especially true for sets presenting specific features that make them easy to manipulate in applications. This easiness is the reason why extreme points of such models (probability intervals, possibility distributions, ...) have been well studied. Yet, imprecise cumulative distributions (a.k.a. p-boxes) constitute an important exception, as the characterization of their extreme points remain to be studied. This is what we do in this paper, where we characterize the maximal number of extreme points of a p-box, give a family of p-boxes that attains this number and show an algorithm that allows to compute the extreme points of a given p-box. To achieve all this, we also provide what we think to be a new characterization of extreme points of a belief function.
\end{abstract}

\section{INTRODUCTION}

Imprecise probability theory [29] is a powerful unifying framework for uncertainty treatment, that uses as basic model of uncertainty a convex probability set $\mathcal{M}$, also called credal set. However, manipulating general credal sets often comes with a high computational price, and in practice simple models [9] are often used in applications. Such models include for instance belief functions, possibility distributions, probability intervals and p-boxes. This latter consists in providing a lower and upper cumulative distribution, extending in a straightforward way cumulative distribution functions. As such, they are instrumental in risk or reliability analysis $[1,4,25]$, in decision making processes based on stochastic orderings [20] or in applications involving naturally ordered spaces such as ordinal regression problems $[2,11]$.

To apply these simpler models, it is important to study their practical aspects, among which is the characterization of the extreme points of the probability sets $\mathcal{M}$ these models induce, when they are defined over finite spaces. Indeed, these extreme points are instrumental to solve computational issues arising in settings such as graphical models [5, 6, 21] or statistical learning [30]. Extreme points of many models ${ }^{1}$ have already been characterized in previous studies. For instance, Dempster [8] shows that the maximal number of extreme points of a belief function on a $n$-element space is $n$ !. It was later [14] proved that the maximal number of extreme points for possibility distributions in a $n$-element space is $2^{n-1}$, and in [23] an algorithm to extract them was provided. In [7], authors studied the extreme

1. University of Oviedo, C/ Federico García Lorca, 18 - 33007 Oviedo, Spain. E-mail address: imontes@uniovi.es Corresponding author. Tel (+34) 985103357, Fax $(+34) 985103354$.

2. Sorbonnes Universités, Université de Technologie de Compiègne, CNRS, UMR 7253 Heudiasyc, 57 Av. de Landshut, 60203 Compiègne E-mail address: sebastien.destercke@hds.utc.fr

${ }^{1}$ To simplify the paper, we will make a small abuse of language and speak of "extreme points of a model" when referring about the extreme points of the credal set induced by such a model. 
points of probability intervals, providing an algorithm to compute them, as well as their maximal number. Extreme points of other specific models such as so-called $\mathrm{k}$-additive measures have also been studied [15], although not necessarily with an imprecise probabilistic interpretation in mind.

However, the extreme points of p-boxes [12] and their generalized version [10] still remain uncharacterised, despite the fact that other aspects such as their connections with existing models or the monotonicity of the lower probability they induce $[13$, $26,27,29$ ] are well-known. The closest existing work we know of explores the shape of extreme cumulative distributions when p-boxes are defined over the continuous real-line [28], yet it does not explore the combinatorial nature of extreme points when p-boxes are defined on finite spaces.

This is the topic of this paper, in which we show that the maximal number of extreme points of a p-box defined on a finite space is a recursive number known as Pell number (Section 4). To do so, we first provide in Section 2 what we believe to be a new characterization of extreme points of belief functions, and then exploit in Section 4 the fact (reminded in Section 3) that p-boxes induce lower probabilities that are belief functions. Finally, Section 5 describes an algorithmic procedure to enumerate the extreme points of the p-boxes, of which the result is a tree whose leaves contain the extreme points.

This paper extends significantly a previous short conference version [18], by providing proofs, examples as well as additional discussions and results.

\section{EXTREME POINTS OF BELIEF FUNCTIONS}

We start this paper by exploring a new way to characterize the extreme points of a belief function, through a set of counting vectors determined by its focal elements. In addition to their own interest, these results will be instrumental to study the extreme points of p-boxes. We first remind the basic definitions of belief functions and their associated credal sets, before proceeding to the characterization of extreme points.

2.1. Basic definitions. Belief functions have been seminally introduced by Dempster [8] and Shafer [24]. They correspond to so-called completely monotone capacities, and can be associated to specific sets of probabilities. Given their attractive mathematical properties, the extreme points of probability sets described by belief functions have already been studied by many authors [3, 8, 23].

A classical means to induce or describe belief functions is through basic probability assignment (bpa). Given a space $\mathcal{X}=\left\{x_{1}, \ldots, x_{n}\right\}$, a basic probability assignment (bpa) is a non-negative function $m: \mathcal{P}(\mathcal{X}) \rightarrow[0,1]$ from the power set $\mathcal{P}(\mathcal{X})$ of $\mathcal{X}$ to the unit interval, satisfying $m(\emptyset)=0$ and $\sum_{B \subseteq \mathcal{X}} m(B)=1$. A bpa $m$ defines a belief Bel and a plausibility $\mathrm{Pl}$ function [24] by:

$$
\operatorname{Bel}(A)=\sum_{B \subseteq A} m(B) \quad \text { and } \quad \operatorname{Pl}(A)=\sum_{B: A \cap B \neq \emptyset} m(B) \quad \forall A \subseteq \mathcal{X} .
$$

These two functions are conjugate since $\operatorname{Bel}(A)=1-\operatorname{Pl}\left(A^{c}\right)$ for any $A \subseteq \mathcal{X}$, hence we can focus on only one of them. A focal set of the belief function Bel is a set $E$ such that $m(E)>0$, and $\mathcal{F}$ will denote the set of focal sets. A belief function induces a credal set

$$
\mathcal{M}(\mathrm{Bel})=\{P \text { Prob. } \mid \operatorname{Bel}(A) \leq P(A) \forall A \subseteq \mathcal{X}\} .
$$

Being closed and convex, the set $\mathcal{M}(\mathrm{Bel})$ can be characterized by its set of extreme points $^{2}$, that we will denote $\mathcal{E} x t(\mathrm{Bel})$. Throughout the paper, and for the sake of

\footnotetext{
${ }^{2}$ Recall that an extreme point $P$ of $\mathcal{M}(\mathrm{Bel})$ is a point such that, if $P_{1}, P_{2} \in \mathcal{M}(\mathrm{Bel})$ and $\alpha P_{1}+(1-\alpha) P_{2}=P$ for some $\alpha \in(0,1)$, then $P_{1}=P_{2}=P$.
} 
simplicity, we will use the expression "extreme points of a belief function" to refer to the extreme points of the credal set induced by the belief function. It is known $[3,8]$ that there is a correspondence between the extreme points of a belief function and the permutations of the elements of $\mathcal{X}$. The extreme point $P_{\sigma} \in \mathcal{E} x t(\mathrm{Bel})$ associated with the permutation $\sigma$ of $\{1, \ldots, n\}$ is given by

$$
\begin{aligned}
P_{\sigma}\left(\left\{x_{\sigma(i)}\right\}\right) & =\operatorname{Bel}\left(\left\{x_{\sigma(i)}, \ldots, x_{\sigma(n)}\right\}\right)-\operatorname{Bel}\left(\left\{x_{\sigma(i+1)}, \ldots, x_{\sigma(n)}\right\}\right) \\
& =\sum_{E \subseteq A_{i}^{\sigma}} m(E)-\sum_{E \subseteq A_{i+1}^{\sigma}} m(E)=\sum_{x_{\sigma(i)} \in E, E \cap A_{i}^{\sigma, C}=\emptyset} m(E)
\end{aligned}
$$

where $A_{i}^{\sigma}=\left\{x_{\sigma(i)}, \ldots, x_{\sigma(n)}\right\}$ and $A_{i}^{\sigma, C}=\left\{x_{\sigma(1)}, \ldots, x_{\sigma(i-1)}\right\}$ is its complement, and where the convention $A_{n+1}^{\sigma}=A_{1}^{\sigma, C}=\emptyset$ is adopted. However, we may have that $P_{\sigma_{1}}=P_{\sigma_{2}}$ for some distinct permutations $\sigma_{1}, \sigma_{2}$, as in general not all permutation give rise to different extreme points, otherwise every belief function would have $n$ ! extreme points. Equation (3b) indicates that building an extreme point comes down, given a permutation $\sigma$, to assign the mass of focal elements $E$ containing $x_{\sigma(1)}$ to $P\left(x_{\sigma(1)}\right)$, remove the corresponding focal elements and then to iterate the procedure until reaching $x_{\sigma(n)}$ or until no focal set remains. Alternatively, we can reinterpret Equation (3b) into a simple pseudo-algorithmic procedure summarised in Algorithm 1, where each focal element $E$ assigns its mass to the first element of $E$ when considering the permutation $\sigma$.

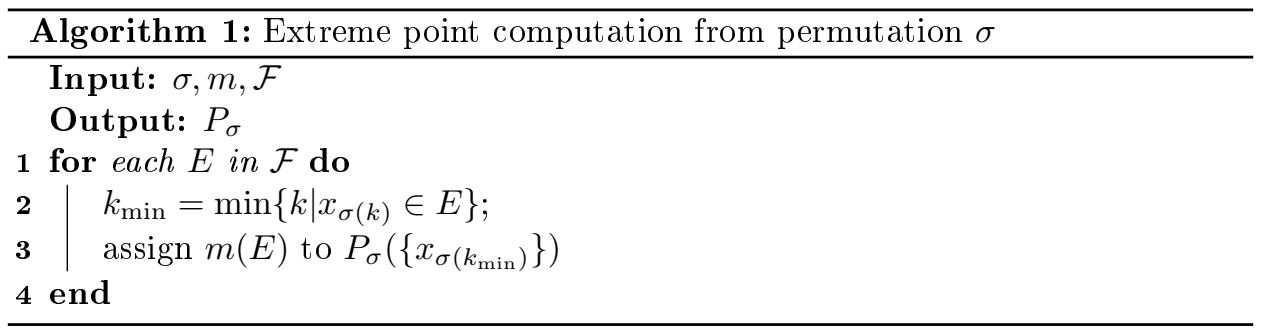

Example 1. Consider a belief function Bel defined on $\mathcal{X}=\left\{x_{1}, x_{2}, x_{3}, x_{4}\right\}$ whose focal sets and their masses are:

\begin{tabular}{c|ccc} 
& $E_{1}=\left\{x_{1}, x_{2}\right\}$ & $E_{2}=\left\{x_{2}, x_{3}, x_{4}\right\}$ & $E_{3}=\left\{x_{3}\right\}$ \\
\hline$m$ & 0.2 & 0.5 & 0.3
\end{tabular}

Consider for example the permutation $\sigma=(1,2,3,4)$. It generates the extreme point $P_{\sigma}=(0.2,0.5,0.3,0)$ in the following way: according to Algorithm $1, m\left(E_{1}\right)$ is assigned to $x_{1}, m\left(E_{2}\right)$ to $x_{2}$ and $m\left(E_{3}\right)$ to $x_{3}$.

If we consider the permutation $\sigma^{\prime}=(1,2,4,3)$, it generates the same extreme point than $\sigma$ because, from Algorithm $1, m\left(E_{1}\right)$ is assigned to $x_{1}$ and $m\left(E_{2}\right)$ to $x_{2}$. Then, the only remaining focal set is $E_{3}=\left\{x_{3}\right\}$, which only can assign its mass to $x_{3}$.

We can already note in Algorithm 1 and Equation (3b) that the structure of the extreme points rely on counting focal elements including specific elements, and not on the numbers $m(E)$. This is formalized in the next section, where we provide a new characterization of belief function extreme points.

2.2. Extreme point characterization. Let us now introduce another way to characterize the extreme points of a belief function. Given a permutation $\sigma$, we denote by $\vec{v}^{\sigma}=\left(v_{1}^{\sigma}, \ldots, v_{n}^{\sigma}\right)$ the vector

$$
\begin{aligned}
v_{i}^{\sigma} & =\mid\left\{E \in \mathcal{F} \mid x_{i} \in E \nexists j<\sigma(i) \text { s.t. } x_{\sigma^{-1}(j)} \in E\right\} \mid \\
& =\left|\left\{E \in \mathcal{F} \mid x_{i} \in E, E \cap\left\{x_{\sigma(1)}, \ldots, x_{\sigma\left(\sigma^{-1}(i)-1\right)}\right\}=\emptyset\right\}\right|,
\end{aligned}
$$


whose $i$ th value is simply the number of focal sets having $x_{i}$ as first element once they are permuted according to $\sigma$. We will also denote by $\mathcal{V}(\mathrm{Bel})$ the set of vectors obtained through Equation (4) for all permutations. We then have the following result.

Proposition 2. Given Bel and two permutations $\sigma_{1}, \sigma_{2}$, we have that $P_{\sigma_{1}}=P_{\sigma_{2}}$ if and only if $\vec{v}^{\sigma_{1}}=\vec{v}^{\sigma_{2}}$.

Proof. Let us first prove that if $P_{\sigma_{1}}=P_{\sigma_{2}}$, then $\vec{v}^{\sigma_{1}}=\vec{v}^{\sigma_{2}}$. For simplicity and without loss of generality, assume that

$$
\begin{aligned}
& \sigma_{1}(1)=1, \ldots, \sigma_{1}(i)=i, \sigma_{1}(i+1)=i+1, \ldots, \sigma_{1}(n)=n \\
& \sigma_{2}(1)=1, \ldots, \sigma_{2}(i)=i, \sigma_{2}(i+1) \neq i+1, \ldots, \sigma_{2}(n)
\end{aligned}
$$

where $i+1$ is the first index for which the two permutations take different values, and we directly have that $v_{j}^{\sigma_{1}}=v_{j}^{\sigma_{2}}$ for $j \leq i$. Let us now show that $v_{i+1}^{\sigma_{1}}=v_{i+1}^{\sigma_{2}}$. Without loss of generality, assume that the focal elements that contain one element among $x_{1}, \ldots, x_{i}$ are $E_{1}, \ldots, E_{\ell}$, and that $E_{\ell+1}, \ldots, E_{s}$ are those that contain $x_{i+1}$ but none of the previous ones. Then

$$
P_{\sigma_{1}}\left(\left\{x_{i+1}\right\}\right)=m\left(E_{\ell+1}\right)+\ldots+m\left(E_{s}\right) .
$$

Now, let us assume that $v_{i+1}^{\sigma_{2}}<v_{i+1}^{\sigma_{1}}$ (the inequality cannot be in the other sense, since $\left.\sigma_{2}^{-1}(i+1)>i+1\right)$ : this means that there is at least one focal element within $E_{\ell+1}, \ldots, E_{s}$ that assigns its mass to an element other than $x_{1}, \ldots, x_{i}, x_{i+1}$, and in this case

$$
P_{\sigma_{2}}\left(x_{i+1}\right)<m\left(E_{\ell+1}\right)+\ldots+m\left(E_{s}\right)=P_{\sigma_{1}}\left(x_{i+1}\right),
$$

which contradicts our initial assumption, hence $v_{i+1}^{\sigma_{2}}=v_{i+1}^{\sigma_{1}}$. We can iterate this reasoning for all $j$, concluding that $\vec{v}^{\sigma_{1}}=\vec{v}^{\sigma_{2}}$.

Let us now prove the converse result, that is if $\vec{v}^{\sigma_{1}}=\vec{v}^{\sigma_{2}}$, then $P_{\sigma_{1}}=P_{\sigma_{2}}$. Throughout this proof, we use the following notation: given a permutation $\sigma$ and $i \in\{1, \ldots, n\}$, define the set $E_{i}^{\sigma}$ as the set of focal sets that assign their masses to the element $x_{i}$ in the extreme point $P_{\sigma}$ :

$$
E_{i}^{\sigma}=\left\{E \in \mathcal{F} \mid x_{i} \in E, \nexists j<\sigma(i) \text { s.t. } x_{\sigma^{-1}(j)} \in E\right\} .
$$

Following this notation, it holds that $\vec{v}_{i}^{\sigma}=\left|E_{i}^{\sigma}\right|$.

Consider now two permutations $\sigma_{1}, \sigma_{2}$ such that $\vec{v}^{\sigma_{1}}=\vec{v}^{\sigma_{2}}$. For the sake of simplicity and without loss of generality, we assume that $\sigma_{1}(i)=i$ for any $i=$ $1, \ldots, n$.

First, let us see that $E_{i}^{\sigma_{1}}=E_{i}^{\sigma_{2}}$ for any $i=1, \ldots, n$. For $i=1$, it holds that:

$$
\begin{aligned}
& E_{1}^{\sigma_{1}}=\left\{E \in \mathcal{F} \mid x_{1} \in E\right\} . \\
& E_{1}^{\sigma_{2}}=\left\{E \in \mathcal{F} \mid x_{1} \in E, \nexists j<\sigma_{2}(1) \text { s.t. } x_{\sigma^{-1}(j)} \in E\right\} \subseteq E_{1} .
\end{aligned}
$$

Since both $E_{1}^{\sigma_{1}}$ and $E_{1}^{\sigma_{2}}$ have the same cardinality (because $\vec{v}_{1}^{\sigma_{1}}=\vec{v}_{1}^{\sigma_{2}}$ ), both sets must coincide.

Assume now that $E_{2}^{\sigma_{1}}=E_{2}^{\sigma_{2}}, \ldots, E_{i-1}^{\sigma_{1}}=E_{i-1}^{\sigma_{2}}$, and let us prove that $E_{i}^{\sigma_{1}}=E_{i}^{\sigma_{2}}$. In this case, these sets can be expressed by:

$$
\begin{aligned}
& E_{i}^{\sigma_{1}}=\left\{E \in \mathcal{F} \mid x_{i} \in E,\left\{x_{1}, \ldots, x_{i-1}\right\} \cap E=\emptyset\right\} . \\
& E_{i}^{\sigma_{2}}=\left\{E \in \mathcal{F} \mid x_{i} \in E, \nexists j<\sigma_{2}(i) \text { s.t. } x_{\sigma^{-1}(j)} \in E\right\} .
\end{aligned}
$$

Let us see that $E_{i}^{\sigma_{2}} \subseteq E_{i}^{\sigma_{1}}$. Firs note that if $E \in E_{i}^{\sigma_{2}}$, then $x_{i} \in E$. Also, assume that there is $j<i$ such that $x_{j} \in E$ (take $j$ the minimum value satisfying this property). Then, $E \in E_{j}^{\sigma_{1}}=E_{j}^{\sigma_{2}}$, and therefore $E \notin E_{i}^{\sigma_{2}}$, a contradiction. Therefore, if $E \in E_{i}^{\sigma_{2}}$, then $x_{i} \in E$ and $\left\{x_{1}, \ldots, x_{i-1}\right\} \cap E=\emptyset$. We conclude that $E_{i}^{\sigma_{2}} \subseteq E_{i}^{\sigma_{1}}$, and also that they coincide because they have the same cardinality (because $\vec{v}_{i}^{\sigma_{1}}=\vec{v}_{i}^{\sigma_{2}}$ ). 
Finally, for any $i=1, \ldots, n$, it holds that:

$$
P_{\sigma_{1}}\left(\left\{x_{i}\right\}\right)=\sum_{E \in E_{i}^{\sigma_{1}}} m(E)=\sum_{E \in E_{i}^{\sigma_{2}}} m(E)=P_{\sigma_{2}}\left(\left\{x_{i}\right\}\right) .
$$

Also note that any vector $\vec{v} \in \mathcal{V}(\mathrm{Bel})$ can be associated with a permutation $\sigma$ generating an extreme point (to see this, note the link between Eq. (4) and Algorithm 1), for instance the permutation having generated it. Since $\mathcal{V}(\mathrm{Bel})$ is in bijection with $\mathcal{E} x t(\mathrm{Bel})$ (any vector is associated with one and only one distinct extreme point), given a vector $\vec{v} \in \mathcal{V}(\mathrm{Bel})$, we can easily determine a permutation generating it by using Algorithm 2.

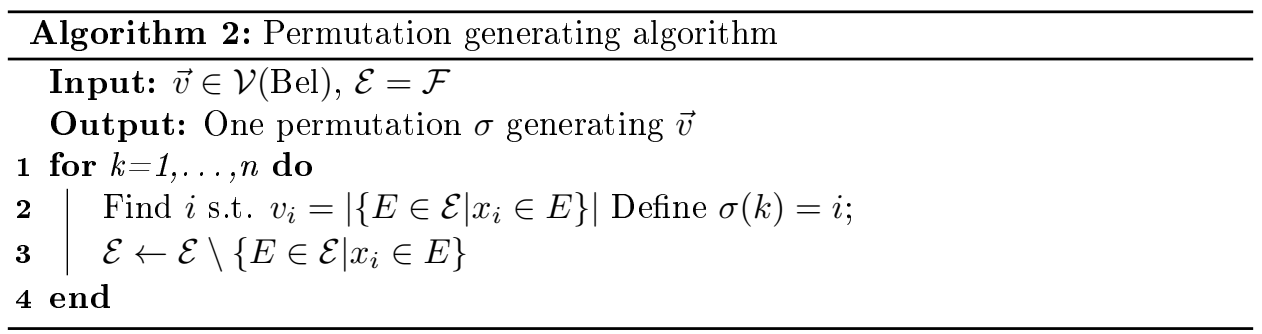

Example 3. Consider a belief function Bel defined on Example 1. We have considered the permutation $\sigma=(1,2,3,4)$, which induces the extreme point $P_{\sigma}=$ $(0.2,0.5,0.3,0)$. Then, $\sigma$ generates the vector:

$$
\vec{v}^{\sigma}=\left(v_{1}^{\sigma}, v_{2}^{\sigma}, v_{3}^{\sigma}, v_{4}^{\sigma}\right)=(1,1,1,0) .
$$

Algorithm 2 can then generate permutations $(1,2,4,3)$ or $(1,2,3,4)$ :

(1) in the first iteration we have $\mathcal{E}=\mathcal{F}$, and only

$$
v_{1}=\left|\left\{E \in \mathcal{F} \mid x_{1} \in E\right\}\right|
$$

satisfies the condition of Line 2 of Algorithm 2, meaning $\sigma(1)=1$;

(2) in the second iteration where $\mathcal{E}=\left\{E_{2}, E_{3}\right\}$, only $v_{2}=1$ satisfy the condition, hence $\sigma(2)=2$ and $\mathcal{E}=\left\{E_{3}\right\}$;

(3) in the third iteration, both $v_{3}=1$ and $v_{4}=0$ satisfy the condition in Line 2 , hence we can have $\sigma(3)=3$ or $\sigma(3)=4$, and the last value of the permutation is the remaining one.

The extreme points of the belief function defined in Example 1, as well as the permutations that generate them, can be seen in Table 1.

Incidentally, this new characterization in terms of "counting" vectors allows us to derive a number of interesting new results about the extreme points of belief functions.

2.3. Some properties of the extreme points of a belief function. We now give some interesting properties about the number of extreme points of belief functions. The first result characterizes the belief functions which have the maximum number of extreme points.

Proposition 4. Let Bel be a belief function on $\mathcal{X}=\left\{x_{1}, \ldots, x_{n}\right\}$. The number of extreme points of Bel is $n$ ! if and only if $\left\{x_{i}, x_{j}\right\}$ is a focal set for any $i, j \in$ $\{1, \ldots, n\}$ such that $i \neq j$. 


\begin{tabular}{|c|c|c|}
\hline Permutation & Probability & $\left(v_{1}^{\sigma}, v_{2}^{\sigma}, v_{3}^{\sigma}, v_{4}^{\sigma}\right)$ \\
\hline $\begin{array}{ll}(1,2,3,4) \quad(1,2,4,3) \\
\end{array}$ & 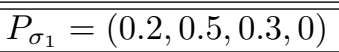 & $\overline{(\overline{(1,1,1,0)}}$ \\
\hline $\begin{array}{c}(1,3,2,4) \quad(1,3,4,2) \quad(3,4,1,2) \\
(3,1,2,4) \quad(3,1,4,2)\end{array}$ & $P_{\sigma_{2}}=(0.2,0,0.8,0)$ & $(1,0,2,0)$ \\
\hline $\begin{array}{c}(1,4,3,2) \quad(1,4,2,3) \quad(4,3,1,2) \\
(4,1,3,2) \quad(4,1,2,3)\end{array}$ & $2,0,0.3,0.5)$ & $(1,0,1,1)$ \\
\hline $\begin{array}{lll}(2,3,1,4) & (2,3,4,1) & (2,4,1,3) \\
(2,1,3,4) & (2,1,4,3) & (2,4,3,1)\end{array}$ & $(0,0.7,0.3,0)$ & $(0,2,1,0)$ \\
\hline $\begin{array}{lll}(3,2,1,4) & (3,2,4,1) & (3,4,2,1)\end{array}$ & $P_{\sigma_{5}}=(0,0.2,0.8,0)$ & $(1,0,2,0)$ \\
\hline $\begin{array}{lll}(4,3,2,1) & (4,2,3,1) & (4,2,1,3)\end{array}$ & $P_{\sigma_{6}}=(0,0.2,0.3,0.5)$ & $(0,1,1,1)$ \\
\hline
\end{tabular}

TABLE 1. Extreme points of the belief function of Example 3.

Proof. Only if: We will proceed by contradiction, showing that if $\left\{x_{i}, x_{j}\right\}$ is not a focal set, then the number of extreme point is lower than $n !$. If the belief function Bel has $n$ ! extreme points, it means that any permutation $\sigma$ of $\{1, \ldots, n\}$ induces a different probability $P_{\sigma}$. Consider two identical permutations $\sigma_{1}$ and $\sigma_{2}$, except for the two last elements, which are such that $\sigma_{1}(n-1)=i, \sigma_{1}(n)=j$ and $\sigma_{2}(n-1)=$ $j, \sigma_{2}(n)=i$. If $\left\{x_{i}, x_{j}\right\}$ is not a focal set, all the non-singleton focal sets including $x_{i}$ or $x_{j}$ would already have assigned their masses to other element $x_{k}(k \neq i, j)$, and therefore $P_{\sigma_{1}}\left(x_{i}\right)=P_{\sigma_{2}}\left(x_{i}\right)$ and $P_{\sigma_{1}}\left(x_{j}\right)=P_{\sigma_{2}}\left(x_{j}\right)$. In that case, we would have $P_{\sigma_{1}}=P_{\sigma_{2}}$, and the belief function Bel could not have more than $n !-1$ extreme points, a contradiction. Hence for every pair $i, j,\left\{x_{i}, x_{j}\right\}$ must be a focal set for Bel to have $n$ ! extreme points .

If: Let us show that if all the sets $\left\{x_{i}, x_{j}\right\}$ are focal sets for any $i, j \in\{1, \ldots, n\}$ $(i \neq j)$, then any two permutations $\sigma_{1} \neq \sigma_{2}$ will generate distinct extreme points. Since $\sigma_{1} \neq \sigma_{2}$, we can assume that:

$$
\sigma_{1}(i) \neq \sigma_{2}(i), \text { but } \sigma_{1}(1)=\sigma_{2}(1), \ldots, \sigma_{1}(i-1)=\sigma_{2}(i-1) .
$$

Let us compute $P_{\sigma_{1}}\left(\left\{x_{\sigma_{1}(i)}\right\}\right)$ and $P_{\sigma_{2}}\left(\left\{x_{\sigma_{1}(i)}\right\}\right)$ :

$$
P_{\sigma_{1}}\left(\left\{x_{\sigma_{1}(i)}\right\}\right)=\sum_{\substack{E \text { focal s.t. } \\ x_{\sigma_{1}(i)} \in E \\ x_{\sigma_{1}(1)}, \ldots, x_{\sigma_{1}(i-1)} \notin E}} m(E) .
$$

Among the focal sets that assign their mass to $x_{\sigma_{1}(i)}$ we find $\left\{x_{\sigma_{1}(i)}, x_{\sigma_{2}(i)}\right\}$. However, when we compute $P_{\sigma_{2}}\left(\left\{x_{\sigma_{1}(i)}\right\}\right)$ we obtain that:

$$
P_{\sigma_{2}}\left(\left\{x_{\sigma_{1}(i)}\right\}\right) \leq \sum_{\substack{E \text { focal s.t. } \\ x_{\sigma_{2}(i)} \in E \\ x_{\sigma_{2}(1)}, \ldots, x_{\sigma_{2}(i-1)} \notin E}} m(E)-m\left(\left\{x_{\sigma_{1}(i)}, x_{\sigma_{2}(i)}\right\}\right)<P_{\sigma_{1}}\left(\left\{x_{\sigma_{1}(i)}\right\}\right),
$$

because by assumption (Eq. (5)), $\sigma_{2}^{-1}\left(\sigma_{1}(i)\right)>i$, and for this reason the non-null mass of the focal set $\left\{x_{\sigma_{1}(i)}, x_{\sigma_{2}(i)}\right\}$ is assigned to $x_{\sigma_{2}(i)}$ and not to $x_{\sigma_{1}(i)}$. Then, we conclude that $P_{\sigma_{1}} \neq P_{\sigma_{2}}$ whenever $\sigma_{1} \neq \sigma_{2}$.

This result is quite surprising, as it tells us that the maximal number of extreme points $n$ ! will be reached only if specific focal elements (those with cardinality two) are present, and that only a quadratic number (i.e., $n(n-1) / 2$ ) of such elements among the $2^{n}$ possible ones are needed to reach this number. The next property tells us that having more focal elements can only increase the number of extreme points, without needing to know the exact mass of the focal elements. 
Proposition 5. Let Bel be a belief function on $\mathcal{X}=\left\{x_{1}, \ldots, x_{n}\right\}$. Denote by $\mathcal{F}$ the set of focal sets of Bel. Let Bel' be another belief function and let $\mathcal{F}^{\prime}=\mathcal{F} \cup\{E\}$ be the set of focal sets of $B e l^{\prime}$, where $E \notin \mathcal{F}$. Then, Bel' has at least as many extreme points as Bel.

Proof. Let us denote by $\left(w_{1}^{\sigma}, \ldots, w_{\sigma}^{n}\right)$ and $\left(v_{1}^{\sigma}, \ldots, v_{\sigma}^{n}\right)$ the vectors associated with $\sigma$ for $\mathrm{Bel}^{\prime}$ and Bel, respectively.

In order to prove this result, it is enough to prove that if two permutations $\sigma_{1}, \sigma_{2}$ give rise to the same extreme point $P_{\sigma_{1}}^{\prime}=P_{\sigma_{2}}^{\prime}$ of $\mathrm{Bel}^{\prime}$, these permutations also give rise to the same extreme point $P_{\sigma_{1}}=P_{\sigma_{2}}$ of Bel.

Consider such two permutations $\sigma_{1}, \sigma_{2}$ that generate the same extreme point of $\mathcal{M}\left(\mathrm{Bel}^{\prime}\right)$. As we have explained, this is equivalent to say that $\left(w_{1}^{\sigma_{1}}, \ldots, w_{n}^{\sigma_{1}}\right)=$ $\left(w_{1}^{\sigma_{2}}, \ldots, w_{n}^{\sigma_{2}}\right)$. Now, we have to see that $\sigma_{1}, \sigma_{2}$ generate the same extreme point of Bel, or equivalently, $\left(v_{1}^{\sigma_{1}}, \ldots, v_{n}^{\sigma_{1}}\right)=\left(v_{1}^{\sigma_{2}}, \ldots, v_{n}^{\sigma_{2}}\right)$.

Let $E=\left\{z_{1}, \ldots, z_{k}\right\}$ be the added focal element, where $z_{1}<\ldots<z_{k}, z_{i} \in$ $\left\{x_{1}, \ldots, x_{n}\right\}$ and $k \leq n$. Let us note that the first element in $E$ must be the same for $\sigma_{1}$ and $\sigma_{2}$, otherwise $E$ would assign its mass to different elements $z_{i}, z_{j}$, and we would have $P_{\sigma_{1}} \neq P_{\sigma_{2}}$. Let $z_{j}$ be this element.

Now, if we remove the focal set $E$, we obtain the vector $\left(v_{1}, \ldots, v_{n}\right)$ given by

$$
v(x)=\left\{\begin{array}{l}
w_{\sigma_{1}}(x)=w_{\sigma_{2}}(x), \text { if } x \neq z_{j} . \\
w_{\sigma_{1}}\left(z_{j}\right)-1=w_{\sigma_{2}}\left(z_{j}\right)-1, \text { if } x=z_{j} .
\end{array}\right.
$$

Then, both $\sigma_{1}$ and $\sigma_{2}$ also generate the same vector $\left(v_{1}, \ldots, v_{n}\right)$ for the focal sets of Bel, and therefore they generate the same extreme point of Bel.

Example 6. Let us continue with the belief function in Example 1. We add a new focal set, $E_{4}=\left\{x_{2}, x_{3}\right\}$, and we modify the masses:

$$
\begin{array}{c|cccc} 
& E_{1}=\left\{x_{1}, x_{2}\right\} & E_{2}=\left\{x_{2}, x_{3}, x_{4}\right\} & E_{3}=\left\{x_{3}\right\} & E_{4}=\left\{x_{2}, x_{3}\right\} \\
\hline m & 0.2 & 0.5 & 0.2 & 0.1
\end{array}
$$

For the belief function in Example 1, both permutations $\sigma=(1,4,2,3)$ and $\sigma=^{\prime}$ $(1,4,3,2)$ generate the same extreme point, $(0.2,0,0.3,0.5)$. However, for the new belief function, their associated counting vectors are different. The counting vector associated with $\sigma$ is $\vec{v}^{\sigma}=(1,1,1,1)$, while the counting vector associated with $\sigma^{\prime}$ is $\vec{v}^{\sigma^{\prime}}=(1,0,2,1)$. Therefore, they induce different extreme points, which are $P_{\sigma}=(0.2,0.1,0.2,0.5)$ and $P_{\sigma^{\prime}}=(0.2,0,0.3,0.5)$.

In general, it can be seen that the belief function in this example has eight different extreme points (and also eight counting vectors), which are summarized in Table 2. This means that adding the new focal set $\left\{x_{2}, x_{3}\right\}$ increases the number of extreme points from six to eight.

Remark 7. Proposition 5 shows that increasing the number of focal sets never decreases the number of extreme points, but does not necessarily increase it. For example, this is the case for a belief function whose focal sets are only all the sets of cardinality two. From Proposition 4 we know that this belief function has $n$ ! extreme points. In this case, we can add as many focal sets as we want (and share the mass between them), the number of extreme points will never be greater than $n !$.

Now that we have established some new ways to look at belief function extreme points, we can connect them to p-boxes and study the extreme points of this latter model. 


\begin{tabular}{c|c|c} 
Permutation & Probability & $\left(v_{1}^{\sigma}, v_{2}^{\sigma}, v_{3}^{\sigma}, v_{4}^{\sigma}\right)$ \\
\hline \hline $\begin{array}{c}(1,2,3,4)(1,2,4,3) \\
(3,2,4)(1,3,4,2)(3,4,1,2)\end{array}$ & $P_{\sigma_{1}}=(0.2,0.5,0.3,0)$ & $(1,1,1,0)$ \\
\hline$(1,4,2,3)(4,1,4,2,3)$ & $P_{\sigma_{2}}=(0.2,0,0.8,0)$ & $(1,0,2,0)$ \\
\hline$(1,4,3,2)(4,1,3,2)(4,3,1,2)$ & $P_{\sigma_{3}}=(0.2,0.1,0.2,0.5)$ & $(1,1,1,1)$ \\
\hline$(2,3,1,4)(2,3,4,1)(2,4,1,3)$ & $P_{\sigma_{4}}=(0.2,0,0.3,0.5)$ & $(1,0,2,1)$ \\
$(2,1,3,4)(2,1,4,3)(2,4,3,1)$ & $P_{\sigma_{5}}=(0,0.7,0.3,0)$ & $(0,2,1,0)$ \\
\hline$(3,2,1,4)(3,2,4,1)(3,4,2,1)$ & $P_{\sigma_{6}}=(0,0.2,0.8,0)$ & $(1,0,2,0)$ \\
\hline$(4,2,1,3)(4,2,3,1)$ & $P_{\sigma_{7}}=(0,0.3,0.2,0.5)$ & $(0,2,1,1)$ \\
\hline$(4,3,2,1)$ & $P_{\sigma_{8}}=(0,0.2,0.3,0.5)$ & $(0,1,2,1)$ \\
\hline
\end{tabular}

TABLE 2. Extreme points of the belief function of Example 6 .

\section{Belief functions and P-BoXes}

To study p-boxes, we will consider from now on that $\mathcal{X}$, in addition to be finite, is an ordered set $\mathcal{X}=\left\{x_{1}, \ldots, x_{n}\right\}$ such that $x_{1}<\ldots<x_{n}$. We will say that a set $E \subseteq \mathcal{X}$ is an interval if any $x \in \mathcal{X}$ between $\min E$ and $\max E$ also belongs to $\mathcal{X}$. We will use the following notation for intervals: $\left[x_{i}, x_{i+k}\right]=\left\{x_{i}, x_{i+1}, \ldots, x_{i+k}\right\}$.

Probability boxes (p-boxes, for short) were introduced in [12]. A p-box, denoted $(\underline{F}, \bar{F})$, is a pair of functions $\underline{F}, \bar{F}: \mathcal{X} \rightarrow[0,1]$ such that $\underline{F}, \bar{F}$ are increasing, $\bar{F}\left(x_{n}\right)=\underline{F}\left(x_{n}\right)=1$ and $\underline{F} \leq \bar{F}$. Here we interpret p-boxes as lower and upper bounds of an imprecisely defined cumulative distribution function. Then, a p-box also defines a credal set

$$
\mathcal{M}(\underline{F}, \bar{F})=\left\{P \text { Prob. } \mid \underline{F}(x) \leq F_{P}(x)=P\left(\left[x_{1}, x\right]\right) \leq \bar{F}(x) \forall x \in \mathcal{X}\right\},
$$

where $F_{P}$ denotes the cumulative distribution function associated with the probability $P$.

It is known that p-boxes are particular instances of belief functions [10], in the sense that for any p-box $(\underline{F}, \bar{F})$ we can define a belief and a plausibility function Bel and Pl such that $\mathcal{M}(\underline{F}, \bar{F})=\mathcal{M}(\mathrm{Bel})$ and

$$
\underline{F}\left(x_{i}\right)=\operatorname{Bel}\left(\left\{x_{1}, \ldots, x_{i}\right\}\right) \text { and } \bar{F}\left(x_{i}\right)=\operatorname{Pl}\left(\left\{x_{1}, \ldots, x_{i}\right\}\right) \forall i=1, \ldots, n .
$$

This belief function can be computed as follows:

$$
\operatorname{Bel}(A)=\min \left\{P(A) \mid \underline{F}(x) \leq F_{P}(x) \leq \bar{F}(x) \forall x \in \mathcal{X}\right\} \quad \forall A \subseteq \mathcal{X},
$$

where again $F_{P}$ denotes the cumulative distribution function associated with the probability $P$.

We can also characterize the belief functions that are equivalent to a p-box (in the sense that for such belief functions Bel, there is a p-box $(\underline{F}, \bar{F})$ such that $\mathcal{M}(\underline{F}, \bar{F})=\mathcal{M}(\mathrm{Bel}))$ in terms of the structure of the focal elements. If we consider the interval order given by

$$
\left[a_{1}, a_{2}\right] \preceq\left[b_{1}, b_{2}\right] \Leftrightarrow a_{1} \leq b_{1}, a_{2} \leq b_{2},
$$

then a belief function Bel whose focal sets $E_{1}, \ldots, E_{k}$ are intervals ordered such that $E_{1} \prec E_{2} \prec \ldots \prec E_{k}$ will be equivalent to a p-box. Conversely, a belief function Bel whose focal sets are intervals ordered according to $\prec$ defines a p-box using Eq. (7), and Bel coincides with the belief function given in Eq. (8), so this property of focal sets is characteristic of p-boxes: a belief function will be a p-box iff it has ordered intervals as focal sets. 


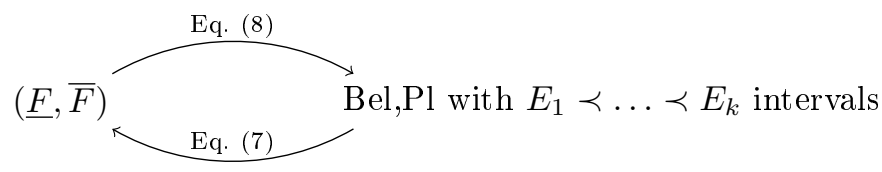

Figure 1. Connection between p-boxes and belief functions.
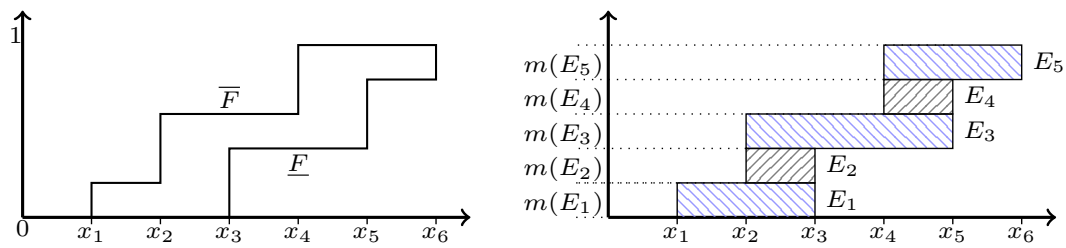

Figure 2. P-box (left) and its associated belief function (right), with focal elements $E_{1}=\left\{x_{1}, x_{2}, x_{3}\right\}, E_{2}=\left\{x_{2}, x_{3}\right\}, E_{3}=$ $\left\{x_{2}, x_{3}, x_{4}, x_{5}\right\}, E_{4}=\left\{x_{4}, x_{5}\right\}$ and $E_{5}=\left\{x_{4}, x_{5}, x_{6}\right\}$.

Moreover, the focal elements can be computed from $(\underline{F}, \bar{F})$ as follows: if there exists $\theta \in[0,1]$ such that

$$
\underline{F}\left(x_{i+1}\right)>\theta \geq \underline{F}\left(x_{i}\right) \text { and } \bar{F}\left(x_{j+1}\right)>\theta \geq \bar{F}\left(x_{j}\right)
$$

for some $i, j \in\{0, \ldots, n-1\}$, where $\underline{F}\left(x_{0}\right)=\bar{F}\left(x_{0}\right)=0$, then $E=\left\{x_{j+1}, x_{j+2}, \ldots, x_{i}\right\}$ is a focal set with mass $m(E)=\min \left\{\underline{F}\left(x_{i+1}\right), \bar{F}\left(x_{j+1}\right)\right\}-\max \left\{\underline{F}\left(x_{i}\right), \bar{F}\left(x_{i}\right)\right\}$. We refer to $[10,13]$ for detailed proofs and algorithms. The connection between p-boxes and belief functions is graphically depicted in Figure 1.

Definition 8. Given a p-box $(\underline{F}, \bar{F})$ defined on $\mathcal{X}$, a set $E$ is called focal set of $(\underline{F}, \bar{F})$ if $E$ is a focal set of the belief function associated with $(\underline{F}, \bar{F})$ by means of Eq. (8).

Example 9. Consider the p-box $(\underline{F}, \bar{F})$ defined on $\mathcal{X}=\left\{x_{1}, \ldots, x_{6}\right\}$ by:

$$
\underline{F}(x)=\left\{\begin{array}{ll}
0 & \text { if } x<x_{3} . \\
0.4 & \text { if } x_{3} \leq x<x_{5} . \\
0.8 & \text { if } x_{5} \leq x<x_{6} . \\
1 & \text { if } x=x_{6} .
\end{array} \quad \bar{F}(x)= \begin{cases}0.2 & \text { if } x=x_{1} . \\
0.6 & \text { if } x_{1}<x \leq x_{4} . \\
1 & \text { if } x>x_{4} .\end{cases}\right.
$$

The focal sets and mass distribution of $(\underline{F}, \bar{F})$ are given by:

$$
\begin{array}{c|ccccc} 
& E_{1} & E_{2} & E_{3} & E_{4} & E_{5} \\
& \left\{x_{1}, x_{2}, x_{3}\right\} & \left\{x_{2}, x_{3}\right\} & \left\{x_{2}, x_{3}, x_{4}, x_{5}\right\} & \left\{x_{4}, x_{5}\right\} & \left\{x_{4}, x_{5}, x_{6}\right\} \\
\hline m & 0.2 & 0.2 & 0.2 & 0.2 & 0.2
\end{array}
$$

Figure 2 provides a picture of the p-box $(\underline{F}, \bar{F})$ and its focal sets.

Possibility measures are another well-known family of plausibility functions. A function $\Pi: \mathcal{X} \rightarrow[0,1]$ is a possibility measure when $\Pi(A)=\sup _{x \in A} \Pi(\{x\})$ for any $A \subseteq \mathcal{X}$. A possibility measure $\Pi$ is a plausibility, and the function $N(A)=1-$ $\Pi\left(A^{c}\right)$, called necessity measure, is a belief function. Since we are dealing with finite sets, the focal sets of a necessity measure, $E_{1}, \ldots, E_{k}$, are nested: $E_{1} \subset \ldots \subset E_{k}$.

The connection between p-boxes and possibility measures has been studied in [27]. We say that a p-box is a possibility measure when its associated belief function is a possibility measure. 

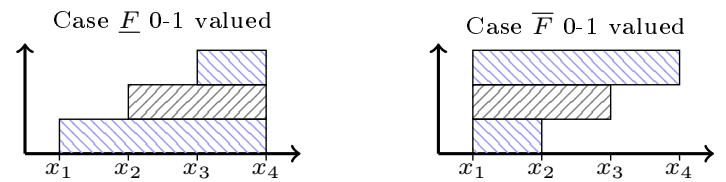

FiguRE 3. Focal sets of the belief function associated with a p-box with $0-1$ valued lower or upper bounds.

\begin{tabular}{c|ccccccc}
$n$ & 1 & 2 & 3 & 4 & 5 & 6 & $\ldots$ \\
\hline $2^{n-1}$ & 1 & 2 & 4 & 8 & 16 & 32 & $\ldots$ \\
$\mathcal{P}_{n}$ & 1 & 2 & 5 & 12 & 29 & 70 & $\ldots$ \\
$n !$ & 1 & 2 & 6 & 24 & 120 & 720 & $\ldots$
\end{tabular}

TABLE 3. First numbers of sequences $2^{n-1}, \mathcal{P}_{n}, n$ !.

Proposition 10 ([27, Cor. 13]). Consider a p-box $(\underline{F}, \bar{F})$ defined in the finite set $\mathcal{X}$. Its associated belief function by means of $E q$. (8) is a possibility measure if and only if either $\underline{F}$ or $\bar{F}$ is $0-1$ valued.

It straightforwardly follows that the focal sets of the possibility measure associated with a p-box are

$$
E_{1}=\left[x_{1}, x_{i_{1}}\right], \quad E_{2}=\left[x_{1}, x_{i_{2}}\right], \quad \ldots \quad E_{k}=\left[x_{1}, x_{i_{k}}\right]=\left[x_{1}, x_{n}\right],
$$

where $x_{1} \leq x_{i_{1}}<\ldots<x_{i_{k}}=x_{n}$, if $\bar{F}$ is $0-1$ valued, or

$$
E_{1}=\left[x_{i_{1}}, x_{n}\right]=\left[x_{1}, x_{n}\right], \quad E_{2}=\left[x_{i_{2}}, x_{n}\right], \quad \ldots \quad E_{k}=\left[x_{i_{k}}, x_{n}\right],
$$

where $x_{1}=x_{i_{1}}<\ldots<x_{i_{k}} \leq x_{n}$, if $\underline{F}$ is $0-1$ valued. This can be graphically seen in Figure 3.

\section{The number of EXTREme Points of A P-Box}

Before studying the extreme points of p-boxes, we need to make a small, useful digression about a specific number sequence: the Pell numbers. Pell numbers form a sequence that follows a recursive relation: $\mathcal{P}_{0}=0, \quad \mathcal{P}_{1}=1, \quad \mathcal{P}_{n}=\mathcal{P}_{n-2}+2 \mathcal{P}_{n-1}$. It is known that $2^{n-1} \leq \mathcal{P}_{n} \leq n$ ! for any $n \geq 1$, and the first numbers associated to these sequences are reminded in Table 3 . As we shall see, it turns out that the maximal number of extreme points of p-boxes on a $n$-element space $\mathcal{X}$ is $\mathcal{P}_{n}$.

4.1. Properties of extreme points of a p-box. Since p-boxes are equivalent to belief functions whose focal elements have a specific structure (are ordered intervals), it is natural to investigate whether the maximal number of extreme points a p-box can have is lower than $n$ !, and in this case what is this maximal number. In order to determine this number, we will first provide a set of useful properties to do so. A first immediate result is the following

Corollary 11. The maximal number of extreme points of a p-box $(\underline{F}, \bar{F})$ on $\mathcal{X}=$ $\left\{x_{1}, \ldots, x_{n}\right\}(n>2)$ lies in the interval $\left[2^{n-1}, n !\right)$.

Proof. When $n>2,\left\{x_{1}, x_{n}\right\}$ cannot be a focal set because the focal sets of a p-box are intervals. Then, according to Proposition $4,(\underline{F}, \bar{F})$ cannot have $n$ ! extreme points.

On the other hand, let us consider the p-box $(\underline{F}, \bar{F})$ such that $\bar{F}=1$ and $\underline{F}$ is strictly increasing $\left(\underline{F}\left(x_{1}\right)<\underline{F}\left(x_{2}\right)<\ldots<\underline{F}\left(x_{n}\right)=1\right)$. From Proposition 10 we know that the belief function associated with $(\underline{F}, \bar{F})$ is a necessity measure, and its 
focal sets are $E_{1}=\left\{x_{1}\right\}, E_{2}=\left\{x_{1}, x_{2}\right\}, \ldots, E_{n}=\left\{x_{1}, \ldots, x_{n}\right\}$. This necessity measure, or its conjugate possibility measure, has the maximal number of extreme points for a necessity measure, $2^{n-1}$. Therefore, we have found a p-box with $2^{n-1}$ extreme points.

Let us now provide properties showing how, starting from an initial p-box, we can incrementally change it into another p-box having a higher number of extreme points.

Proposition 12. Let $(\underline{F}, \bar{F})$ be a p-box on $\mathcal{X}=\left\{x_{1}, \ldots, x_{n}\right\}$ and denote by Bel its associated belief function, and by $\mathcal{F}$ the set of focal sets of Bel. Assume that the following sets are focal sets:

$$
E^{-}=\left[x_{i}, x_{j}\right], \quad E=\left[x_{i}, x_{j+1}\right], \quad E^{+}=\left[x_{i+1}, x_{j+1}\right],
$$

for $j \geq i+2$. Denote by Bel' a belief function whose set of focal sets $\mathcal{F}^{\prime}$ is given by $\mathcal{F}^{\prime}=(\mathcal{F} \backslash\{E\}) \cup E^{*}$, where $E^{*}=\left[x_{i+1}, x_{j}\right]$.

(1) Bel is a belief function associated with a $p$-box $\left(\underline{F}^{\prime}, \bar{F}^{\prime}\right)$.

(2) $\left(\underline{F^{\prime}}, \bar{F}^{\prime}\right)$ has at least as many extreme points as $(\underline{F}, \bar{F})$.

(3) $\left(\underline{F^{\prime}}, \bar{F}\right)$ has more extreme points than $(\underline{F}, \bar{F})$ only if $j>i+2$.

Proof. (1): First of all, note that $E^{-} \prec E^{*} \prec E^{+}$, so these three focal sets are ordered according to $\prec$. Furthermore, the remaining focal sets are also ordered because they are the focal sets of the p-box $(\underline{F}, \bar{F})$, and because $E$ and $E^{*}$ are the only two focal sets between $E^{-}$and $E^{+}$according to $\prec$. Then, we conclude that the focal sets of the belief function $\mathrm{Bel}^{\prime}$ are intervals and they are ordered with respect to $\prec$, which implies that it is the belief function associated with a p-box $\left(\underline{F^{\prime}}, \bar{F}^{\prime}\right)$.

$(2)+(3)$ : Let us now show that $\left|\mathcal{V}\left(\mathrm{Bel}^{\prime}\right)\right| \geq|\mathcal{V}(\mathrm{Bel})|$. Given a permutation $\sigma$, denote by $w^{\sigma}$ and $v^{\sigma}$ the vectors in $\left|\mathcal{V}\left(\mathrm{Bel}^{\prime}\right)\right|$ and $|\mathcal{V}(\mathrm{Bel})|$ generated by $\sigma$, and by $x_{*}$ the element in $E^{*}=\left[x_{i+1}, x_{j}\right]$ that first appears in $\sigma$, meaning that $\sigma^{-1}(*) \leq$ $\min \left(\sigma^{-1}(i+1), \sigma^{-1}(j)\right)$. Note also that, since $E^{*} \subset E, x_{*} \in E$.

Since the only change between $\mathrm{Bel}$ and $\mathrm{Bel}^{\prime}$ is the replacement of $E$ by $E^{*}$, distinction between extreme points of Bel and $\mathrm{Bel}^{\prime}$ only depends on the relative positions of $x_{*}, x_{i}$ and $x_{j+1}$ in a permutation. There are then two cases (forming a partition of every possible permutations).

Case 1: if $\sigma^{-1}(*) \nsupseteq \max \left(\sigma^{-1}(i), \sigma^{-1}(j+1)\right)$, then there is a bijection between $w^{\sigma}$ and $v^{\sigma}$, more precisely

$$
w^{\sigma}=v^{\sigma} \text { if } \sigma^{-1}(*) \leq \max \left(\sigma^{-1}(i), \sigma^{-1}(j+1)\right)
$$

since in this case both $m(E)$ and $m\left(E^{*}\right)$ are assigned to $x_{*}$. If $\sigma^{-1}(i) \leq$ $\sigma^{-1}(*) \leq \sigma^{-1}(j+1)$, then

$$
v^{\sigma}(x)=\left\{\begin{array}{l}
w^{\sigma}\left(x_{i}\right)+1, \quad \text { if } x=x_{i} . \\
w^{\sigma}\left(x_{*}\right)-1, \quad \text { if } x=x_{*} . \\
w^{\sigma}(x), \quad \text { otherwise. }
\end{array}\right.
$$

since $m(E)$ is assigned to $x_{i}$ and $m\left(E^{*}\right)$ to $x_{*}$. The situation $\sigma^{-1}(j+1) \leq$ $\sigma^{-1}(*) \leq \sigma^{-1}(i)$ is similar. Let us denote by $k_{1}$ the number of distinct vectors in both $\left|\mathcal{V}\left(\mathrm{Bel}^{\prime}\right)\right|$ and $|\mathcal{V}(\mathrm{Bel})|$ corresponding to this situation.

Case 2: Consider now a permutation with $\sigma^{-1}(*) \geq \max \left(\sigma^{-1}(i), \sigma^{-1}(j+\right.$ 1)) and the associated $w^{\sigma}, v^{\sigma}$, meaning that $m(E)$ is assigned to $x_{k}$ with $k=\arg _{i, j+1} \min \left(\sigma^{-1}(i), \sigma^{-1}(j+1)\right)$, and $m\left(E^{*}\right)$ to $x_{*}$. Now, consider the following: 

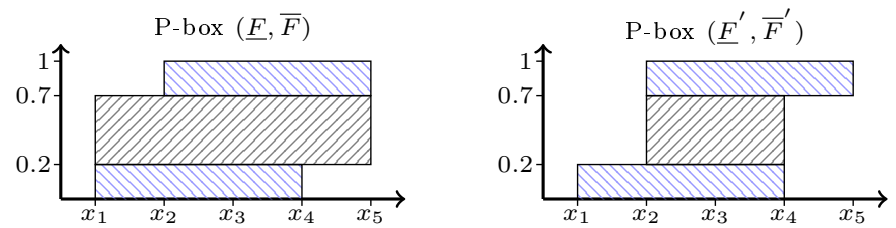

Figure 4. P-boxes $(\underline{F}, \bar{F})$ and $\left(\underline{F}^{\prime}, \bar{F}^{\prime}\right)$ of Example 13.

- on one hand, if we consider the permutation $\sigma^{\prime}$ such that $\sigma^{\prime}(k)=\sigma(k)$ for any $k \neq i, j+1$, and $\sigma^{\prime}(i)=\sigma(j+1), \sigma^{\prime}(j+1)=\sigma(i)$, then we have $v^{\sigma} \neq v^{\sigma^{\prime}}$, but $w^{\sigma}=w^{\sigma^{\prime}}$

- on the other hand, we can consider $j-i$ (the number of elements of $E^{*}$ ) permutations $\sigma^{\prime}$ where we exchange in the permutation $\sigma$ the indices of $x_{*}$ and of another element of $E^{*}$, for which $v^{\sigma}=v^{\sigma^{\prime}}$, but $w^{\sigma} \neq w^{\sigma^{\prime}}$.

This means that for a permutation satisfying $\sigma^{-1}(*) \geq \max \left(\sigma^{-1}(i), \sigma^{-1}(j+1)\right)$, we can construct one additional permutation satisfying this constraint and generating 2 distinct vectors of $|\mathcal{V}(\mathrm{Bel})|$ whose permutation will give the same vector of $\left|\mathcal{V}\left(\mathrm{Bel}^{\prime}\right)\right|$, and construct $j-i$ additional permutations satisfying this constraint and generating distinct vectors of $|\mathcal{V}(\mathrm{Bel})|$ but giving the same vector of $\left|\mathcal{V}\left(\mathrm{Bel}^{\prime}\right)\right|$. Let $k_{2}$ be the quantity of such initial permutations. Then we have

$$
|\mathcal{V}(\mathrm{Bel})|=\mathrm{k}_{1}+2 \mathrm{k}_{2} \geq \mathrm{k}_{1}+(\mathrm{j}-\mathrm{i}) \mathrm{k}_{2}=\left|\mathcal{V}\left(\mathrm{Bel}^{\prime}\right)\right|
$$

with the inequality being strict only if $j-i>2$.

Example 13. Consider the p-box $(\underline{F}, \bar{F})$ on $\left\{x_{1}, x_{2}, x_{3}, x_{4}, x_{5}\right\}$ whose focal sets are:

$$
E_{1}=\left\{x_{1}, x_{2}, x_{3}, x_{4}\right\}, \quad E_{2}=\left\{x_{1}, x_{2}, x_{3}, x_{4}, x_{5}\right\}, \quad E_{3}=\left\{x_{2}, x_{3}, x_{4}, x_{5}\right\}
$$

with $m\left(E_{1}\right)=0.2, m\left(E_{2}\right)=0.5$ and $m\left(E_{3}\right)=0.3$. Also, consider the p-box $\left(\underline{F}^{\prime}, \bar{F}^{\prime}\right)$ whose focal sets are:

$$
E_{1}=\left\{x_{1}, x_{2}, x_{3}, x_{4}\right\}, \quad E_{2}^{*}=\left\{x_{2}, x_{3}, x_{4}\right\}, \quad E_{3}=\left\{x_{2}, x_{3}, x_{4}, x_{5}\right\},
$$

with the same masses $m^{\prime}\left(E_{1}\right)=0.2, m^{\prime}\left(E_{2}^{*}\right)=0.5$ and $m^{\prime}\left(E_{3}\right)=0.3$. These p-boxes are depicted in Figure 4.

Using the notation of the previous proposition, we have $x_{i}=x_{1}, x_{i+1}=x_{2}$, $x_{j}=x_{4}$ and $x_{j+1}=x_{5}$. The permutations and vectors $w$ that generates the extreme points of $\mathrm{Bel}^{\prime}$ are the following:

\begin{tabular}{ccccc} 
& Case & Permutation & Vector $w$ & Probability \\
\hline \hline \multirow{6}{*}{$k_{1}=9$} & Case 1 & $(2,1,3,4,5)$ & $(0,3,0,0,0)$ & $(0,1,0,0,0)$ \\
& Case 1 & $(3,1,2,4,5)$ & $(0,0,3,0,0)$ & $(0,0,1,0,0)$ \\
& Case 1 & $(4,1,2,3,5)$ & $(0,0,0,3,0)$ & $(0,0,0,1,0)$ \\
& Case 1 & $(1,2,3,4,5)$ & $(1,2,0,0,0)$ & $(0.2,0.8,0,0,0)$ \\
& Case 1 & $(1,3,2,4,5)$ & $(1,0,2,0,0)$ & $(0.2,0,0.8,0,0)$ \\
& Case 1 & $(1,4,2,3,5)$ & $(1,0,0,2,0)$ & $(0.2,0,0,0.8,0)$ \\
& Case 1 & $(5,2,1,3,4)$ & $(0,2,0,0,1)$ & $(0,0.7,0,0,0.3)$ \\
& Case 1 & $(5,3,1,2,4)$ & $(0,0,2,0,1)$ & $(0,0,0.7,0,0.3)$ \\
& Case 1 & $(5,4,1,2,3)$ & $(0,0,0,2,1)$ & $(0,0,0,0.7,0.3)$ \\
\hline \multirow{5}{*}{$(j-i) k_{2}=3$} & Case 2 & $(1,5,2,3,4)$ & $(1,1,0,0,1)$ & $(0.2,0.5,0,0,0.3)$ \\
& Case 2 & $(1,5,3,2,4)$ & $(1,0,1,0,1)$ & $(0.2,0,0.5,0,0.3)$ \\
& Case 2 & $(1,5,4,2,3)$ & $(1,0,0,1,1)$ & $(0.2,0,0,0.5,0.3)$ \\
\hline
\end{tabular}


where for the second situation, $\sigma=(1,5,2,3,4)$ and other vectors are obtained by permuting 2 with either 3 or 4 . Thus, $\mathcal{M}\left(\mathrm{Bel}^{\prime}\right)$ has 12 extreme points. On the other hand, the extreme points of Bel are the following:

\begin{tabular}{ccccc} 
& Case & Permutation & Vector $v$ & Probability \\
\hline \hline \multirow{6}{*}{$k_{1}=9$} & Case 1 & $(2,1,3,4,5)$ & $(0,3,0,0,0)$ & $(0,1,0,0,0)$ \\
& Case 1 & $(3,1,2,4,5)$ & $(0,0,3,0,0)$ & $(0,0,1,0,0)$ \\
& Case 1 & $(4,1,2,3,5)$ & $(0,0,0,3,0)$ & $(0,0,0,1,0)$ \\
& Case 1 & $(1,2,3,4,5)$ & $(2,1,0,0,0)$ & $(0.7,0.3,0,0,0)$ \\
& Case 1 & $(1,3,2,4,5)$ & $(2,0,1,0,0)$ & $(0.7,0,0.3,0,0)$ \\
& Case 1 & $(1,4,2,3,5)$ & $(2,0,0,1,0)$ & $(0.7,0,0,0.3,0)$ \\
& Case 1 & $(5,2,1,3,4)$ & $(0,1,0,0,2)$ & $(0,0.2,0,0,0.8)$ \\
& Case 1 & $(5,3,1,2,4)$ & $(0,0,1,0,2)$ & $(0,0,0.2,0,0.8)$ \\
& Case 1 & $(5,4,1,2,3)$ & $(0,0,0,1,2)$ & $(0,0,0,0.2,0.8)$ \\
\hline \multirow{2}{*}{$2 \cdot k_{2}=2$} & Case 2 & $(1,5,2,3,4)$ & $(2,0,0,0,1)$ & $(0.7,0,0,0,0.3)$ \\
& Case 2 & $(5,1,2,3,4)$ & $(1,0,0,0,2)$ & $(0.2,0,0,0,0.8)$ \\
\hline
\end{tabular}

As we can see, in the first situation we obtain the same number $\left(k_{1}=9\right)$ of extreme points. In the second situation, the permutations $(1,5,2,3,4)$ and $(5,1,2,3,4)$ generate the same extreme point for Bel', but different for Bel. Furthermore, permutations $(1,5,2,3,4),(1,5,3,2,4)$ and $(1,5,4,2,3)$ give rise to the same extreme point for Bel, while for $\mathrm{Bel}^{\prime}$ they generate three different extreme points because the mass of $E_{2}^{*}$ goes to $x_{2}, x_{3}$ or $x_{4}$, respectively. The same happens with the permutations $(5,1,2,3,4),(5,1,3,2,4)$ and $(5,1,4,2,3)$.

So in the end, Bel $^{\prime}$ has $k_{1}+3 k_{2}=12$ extreme points while Bel only has $k_{1}+2 k_{2}=$ 11 extreme points.

Finally, we adapt the results from Subsection 2.3 to the specific case of p-boxes. The first result is somewhat similar to Proposition 12, who was telling us when we can replace a focal element $E$ by another $E^{*} \subset E$ to increase the number of extreme points.

Corollary 14. Let $(\underline{F}, \bar{F})$ be a p-box on $\left\{x_{1}, \ldots, x_{n}\right\}$ and denote by Bel its associated belief function. Let $\mathcal{F}$ denote the set of focal sets of Bel, and assume that $E_{k}=\left\{x_{i}\right\} \in \mathcal{F}$ for some $i=2, \ldots, n-1$. Consider a belief function $\mathrm{Bel}^{\prime}$ whose set of focal sets is given by $\mathcal{F}^{\prime}=\left(\mathcal{F} \backslash\left\{x_{i}\right\}\right) \cup E_{k}^{*}$, where $E_{k}^{*}=\left\{x_{i-1}, x_{i}, x_{i+1}\right\}$. Then, $\mathrm{Bel}^{\prime}$ is also a p-box and $\mathrm{Bel}^{\prime}$ has strictly more extreme points than Bel.

Proof. The fact that $\mathrm{Bel}^{\prime}$ is a p-box follows from the fact that if $E_{k}=\left\{x_{i}\right\}$, then $E_{k-1}$ satisfies max $E_{k-1} \leq x_{i}$ and $\min E_{k-1} \leq x_{i-1}$ and $E_{k+1}$ satisfies min $E_{k+1} \geq$ $x_{i}$ and $\max E_{k+1} \geq x_{i+1}$, hence we still have $E_{k-1} \prec E_{k}^{*} \prec E_{k+1}$ and the focal sets of Bel' $^{\prime}$ are still ordered. The second part is direct since for any permutation $m\left(E_{k}\right)$ can only go to $x_{i}$, while this is not the case for $m\left(E_{k}^{*}\right)$.

The second result builds upon Proposition 5, and shows that adding non-singleton focal sets, in the case of p-boxes, strictly increases the number of extreme points.

Proposition 15. Let $(\underline{F}, \bar{F})$ be a p-box on $\left\{x_{1}, \ldots, x_{n}\right\}$ and denote by Bel its associated belief function, and let $E_{1} \prec \ldots \prec E_{k}$ be its focal elements. If there exists an interval $E=\left[x_{i}, x_{j}\right]$, with $i<j$, such that $E_{l} \prec E \prec E_{l+1}$ for some $i=1, \ldots, n-1$, then the $p$-box $\left(\underline{F}^{\prime}, \bar{F}^{\prime}\right)$ whose focal elements are $E_{1}, \ldots, E_{k}, E$ has more focal sets than $(\underline{F}, \bar{F})$ and has more extreme points than $(\underline{F}, \bar{F})$.

Proof. That it has at least as many extreme points is direct using Proposition 5, and we now have to prove that it has strictly more. 
If $|E|=2$, the result can be proved by using the same arguments as in Proposition 4. If $|E|>2$, let us consider the two following permutations

$$
\sigma_{1}=(i-1, i-2, \ldots, 1, j+1, j+2, \ldots, n, i, j, i+1, i+2, \ldots, j-2, j-1)
$$

and

$$
\sigma_{2}=(j+1, j+2, \ldots, n, i-1, i-2, \ldots, 1, j, i, i+1, i+2, \ldots, j-2, j-1)
$$

that will generate the same vectors and extreme points for Bel. To see this, consider that

- any focal set preceding $E$ in the order will necessarily contain an element within $x_{1}, \ldots, x_{i}$ and have an upper bound with an index being at most $j$, and that if it contains $x_{i}$, its upper bound index is necessarily lower than $j$ and that,

- any focal set succeeding $E$ will necessarily contain an element within $x_{j}$, $\ldots, x_{n}$ and have a lower bound with an index being at least $i$, and that if it contains $x_{j}$, its lower bound index is necessarily higher than $i$.

Furthermore, the focal sets preceding $E$ and succeeding $E$ form the partition $\left\{E_{1}, \ldots, E_{l}\right\} \cup\left\{E_{l+1}, \ldots, E_{k}\right\}$ of $E_{1}, \ldots, E_{k}$. This means that no set in $E_{1}, \ldots, E_{k}$ can contain both $x_{i}$ and $x_{j}$. Also note that, if we denote $v^{\sigma}$ the vector of $\mathcal{V}(\mathrm{Bel})$ obtained for $\sigma$, we have $v^{\sigma}(i+1)=v^{\sigma}(i+2)=\ldots=v^{\sigma}(j-2)=v^{\sigma}(j-1)=0$ for $\sigma=\sigma_{1}$ and $\sigma=\sigma_{2}$ (otherwise $E$ could not be inserted in the existing focal sets).

To finish the proof, it is then sufficient to note that $\sigma_{1}$ and $\sigma_{2}$ would generate two distinct vectors of $\mathcal{V}\left(\mathrm{Bel}^{\prime}\right)$, since $m(E)$ would be assigned to $x_{i}$ for $\sigma_{1}$, and to $x_{j}$ for $\sigma_{2}$, as $E$ contains both $x_{i}$ and $x_{j}$

This Proposition also tells us that, to have a maximal number of extreme points, a p-box should be such that if $E_{i}=\left[x_{k}, x_{l}\right]$ is a focal set, then $E_{i+1}$ should be either $\left[x_{k+1}, x_{l}\right]$ or $\left[x_{k}, x_{l+1}\right]$, that is the p-box focal sets should be obtained by iteratively incrementing their lower/upper bound by one element, so that they have a maximal number of them.

4.2. The Pell family of p-boxes. Let us now show that a particular subset of p-boxes, that we will call the Pell family, and its members Pell p-boxes, have as number of extreme points the Pell number. We will then proceed to show that these p-boxes are actually the only one having the maximal number of extreme points a p-box can have.

The family of Pell p-boxes consists of those p-boxes on $\mathcal{X}=\left\{x_{1}, \ldots, x_{n}\right\}$ such that the following sets are focal:

$$
\begin{aligned}
& \left\{x_{1}, x_{2}\right\},\left\{x_{n-1}, x_{n}\right\}, \\
& \forall i=2, \ldots, n-1,\left\{x_{i-1}, x_{i}, x_{i+1}\right\}, \\
& \forall i=2, \ldots, n-1, \text { either }\left[x_{i-1}, x_{i+2}\right] \text { or }\left[x_{i}, x_{i+1}\right] .
\end{aligned}
$$

The sets $\left\{x_{1}\right\}$ and $\left\{x_{n}\right\}$ are also allowed to be focal; therefore, we assume throughout this paragraph that $m\left(\left\{x_{1}\right\}\right), m\left(\left\{x_{n}\right\}\right) \geq 0$. However, the mass of the remaining sets is strictly positive. In Figure 5 we have depicted all the p-boxes of the Pell family for $n=4$ and $n=5$. Note that Pell p-boxes are characterized by the fact that their focal sets have a maximal cardinality of 4 .

Let us first start by showing that some members of the Pell family indeed have a number of extreme points equal to the Pell number. 


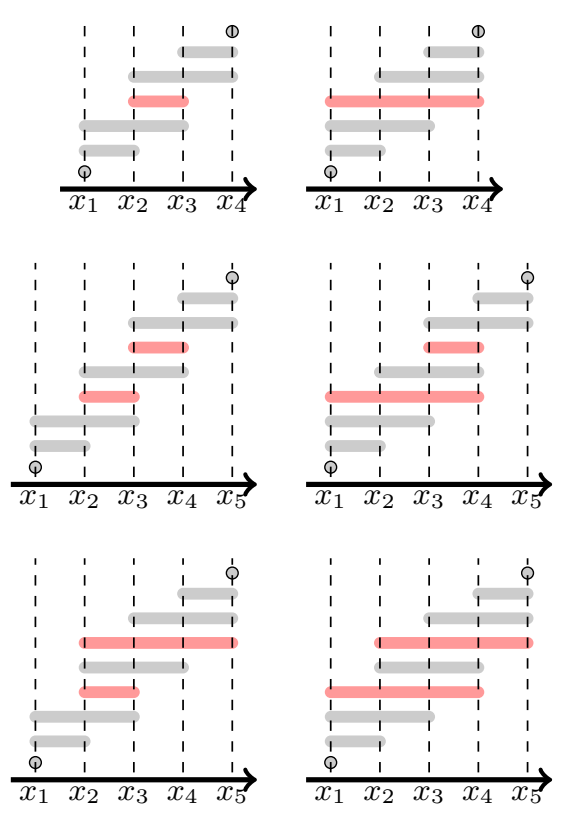

Figure 5. P-boxes of the Pell family for $n=4$ and $n=5$.

Proposition 16. Consider the p-box $(\underline{F}, \bar{F})$ of the Pell family such that the following sets are focal:

$$
\begin{aligned}
& \left\{x_{1}, x_{2}\right\}, \quad\left\{x_{n-1}, x_{n}\right\}, \\
& \left\{x_{i-1}, x_{i}, x_{i+1}\right\} \quad \forall i=2, \ldots, n-1, \\
& {\left[x_{i}, x_{i+1}\right] \quad \forall i=2, \ldots, n-1 .}
\end{aligned}
$$

Also, we assume that $m\left(\left\{x_{1}\right\}\right), m\left(\left\{x_{n}\right\}\right) \geq 0$. Then, the number of extreme points of $(\underline{F}, \bar{F})$ is the Pell number $\mathcal{P}_{n}$.

Proof. We will proceed by induction over $n$. For $n=1$, the result is trivial. Assuming now that the results is true for $n-1$, let us consider the case of $n$. Assume that $m\left(\left\{x_{1}\right\}\right)>0$; the case $m\left(\left\{x_{1}\right\}\right)=0$ follows by analogy. First note that $v_{1}^{\sigma} \in\{1,2,3\}$ whatever the permutation, as the only focal sets including $x_{1}$ are $\left\{x_{1}\right\},\left\{x_{1}, x_{2}\right\},\left\{x_{1}, x_{2}, x_{3}\right\}$ by hypothesis. In the case $m\left(\left\{x_{1}\right\}\right)=0, v_{1}^{\sigma} \in\{0,1,2\}$, and the proof is completely analogous.

Let us count the number of vectors $v^{\sigma}$ such that $v_{1}^{\sigma}=3$. This means that $\left\{x_{1}, x_{2}\right\}$ and $\left\{x_{1}, x_{2}, x_{3}\right\}$ assign their mass to $x_{1}$. For this, $x_{1}$ necessarily appears before $x_{2}$ and $x_{3}$ in the permutation $\sigma$ associated with $v^{\sigma}$. Furthermore, since $x_{1}$ has no common focal sets with $x_{4}, \ldots, x_{n}$, the position of $x_{1}$ w.r.t. $x_{4}, \ldots, x_{n}$ is irrelevant. Therefore, we can consider that $x_{1}$ appears in the first position in the permutation $\sigma$ associated with $v^{\sigma}$. Then, the number of different vectors $v^{\sigma}$ such that $v_{1}^{\sigma}=3$ equals the number of different combinations of $x_{2}, \ldots, x_{n}$ generating different extreme points, which by hypothesis of induction equals $\mathcal{P}_{n-1}$.

Let us now count the number of vectors $v^{\sigma}$ such that $v_{1}^{\sigma}=2$. This means that $x_{3}$ appears before $x_{1}$, and $x_{1}$ appears before $x_{2}$ in the permutation $\sigma$ associated with $v^{\sigma}$. Again, since $x_{1}$ has no common focal sets with $x_{4}, \ldots, x_{n}$, we can assume that the two last elements in the permutation $\sigma$ associated with $v^{\sigma}$ are $x_{\sigma(n-1)}=$ $x_{1}, x_{\sigma(n)}=x_{2}$. Then, the number of vectors $v^{\sigma}$ such that $v_{1}^{\sigma}=2$ equals the number of combinations of the elements $x_{3}, \ldots, x_{n}$, which by hypothesis of induction equals $\mathcal{P}_{n-2}$. 
Finally, let us count the number of vectors $v^{\sigma}$ such that $v_{1}^{\sigma}=1$. This means that both $x_{2}$ and $x_{3}$ appear before $x_{1}$ in the permutation $\sigma$ associated with $v^{\sigma}$. Using again the fact that $x_{1}$ has no common focal set with $x_{4}, \ldots, x_{n}$, so we can assume that $x_{1}$ appears in the last position of the permutation: $x_{\sigma(n)}=x_{1}$. Then, the number of vectors such that $v_{1}^{\sigma}=1$ is the number of different combinations of $x_{2}, \ldots, x_{n}$, which equals $\mathcal{P}_{n-1}$.

Then, the number of extreme points coincide with the sum of vectors such that $v_{1}^{\sigma}$ equals 1,2 or $3: 2 \mathcal{P}_{n-1}+\mathcal{P}_{n-2}=\mathcal{P}_{n}$, the Pell number.

In fact, we can use Propositions 12 and 16 to prove that all the p-boxes of the Pell family have $\mathcal{P}_{n}$ extreme points.

Theorem 17. If $(\underline{F}, \bar{F})$ is a p-box of the Pell family on $\mathcal{X}=\left\{x_{1}, \ldots, x_{n}\right\}$, its number of extreme points is the Pell number $\mathcal{P}_{n}$.

Proof. Consider a p-box $(\underline{F}, \bar{F})$ of the Pell family. If $\left[x_{i}, x_{i+1}\right]$ is a focal set for any $i=2, \ldots, n$, we can apply Proposition 16 directly to get the result. Assume that this is not the case, and denote by $x_{i_{1}}<\ldots<x_{i_{k}}$ the elements such that $2 \leq i_{1}<\ldots<i_{k}<n-1$ and the focal set is $\left[x_{i_{j}-1}, x_{i_{j}+2}\right]$, for $j=1, \ldots, k$.

Applying Proposition 12 with the sets

$$
\begin{aligned}
& E^{-}=\left\{x_{i_{1}-1}, x_{i_{1}}, x_{i_{1}+1}\right\}, \quad E^{+}=\left\{x_{i_{1}}, x_{i_{1}+1}, x_{i_{1}+2}\right\}, \\
& E=\left\{x_{i_{1}-1}, x_{i_{1}}, x_{i_{1}+1}, x_{i_{1}+2}\right\} \text { and } E^{*}=\left\{x_{i_{1}}, x_{i_{1}+1}\right\}
\end{aligned}
$$

we obtain a new p-box that has the same number of focal sets than $(\underline{F}, \bar{F})$, since we substitute the focal set $E$ by $E^{*}$ and $i_{1}+1-\left(i_{1}-1\right)=2$. Again, we can do the same for $j=2, \ldots, k$, keeping the same number of extreme points, and at the end we will obtain a p-box described by Proposition 16, whose number of extreme points is $\mathcal{P}_{n}$.

4.3. Maximal number of extreme points of a p-box. Using the tools we have developed so far, we can now establish the main result of the paper, that is characterizing the maximal number of extreme points of a p-box, and that this number is reached only for specific structures.

Theorem 18. Let $(\underline{F}, \bar{F})$ be a p-box on $\left\{x_{1}, \ldots, x_{n}\right\}$. The following holds:

(1) The maximal number of extreme points of a $p$-box on $\left\{x_{1}, \ldots, x_{n}\right\}$ is the Pell number $\mathcal{P}_{n}$.

(2) $(\underline{F}, \bar{F})$ has $\mathcal{P}_{n}$ extreme points if and only if it is a p-box of the Pell family.

Proof. The idea of the proof is simple: starting with an arbitrary p-box $(\underline{F}, \bar{F})$, we will increase its number of focal sets and extreme points to reach a p-box of the Pell family, through the mechanisms we have developed so far.

The first step is to remove unnecessary singletons in the following way: if $E_{i}=$ $\left\{x_{i}\right\}$ is a focal set of $(\underline{F}, \bar{F})$ for some $i=2, \ldots, n-1$, we substitute it by $E_{i}^{*}=$ $\left\{x_{i-1}, x_{i}, x_{i-1}\right\}$. We consider the p-box $\left(\underline{F}_{1}, \bar{F}_{1}\right)$ by considering the non-singleton focal sets of $(\underline{F}, \bar{F})$ plus the elements $E_{i}^{*}$ such that $\left\{x_{i}\right\}$ is a focal set. According to Corollary $14,\left(\underline{F}_{1}, \bar{F}_{1}\right)$ has at least as many points as $(\underline{F}, \bar{F})$, and strictly more if one singleton focal set has been replaced.

The second step consists in adding focal sets to fill in "gaps" between two consecutive focal sets of $\left(\underline{F}_{1}, \bar{F}_{1}\right)$ (thus non-decreasing the number of extreme points), building a second p-box $\left(\underline{F}_{2}, \bar{F}_{2}\right)$. Denote by $E_{1} \prec \ldots \prec E_{k}$ the focal sets of $\left(\underline{F}_{1}, \bar{F}_{1}\right)$. Let us use the following notation:

$$
\underline{e}_{i}=\min E_{i} \text { and } \bar{e}_{i}=\max E_{i}
$$




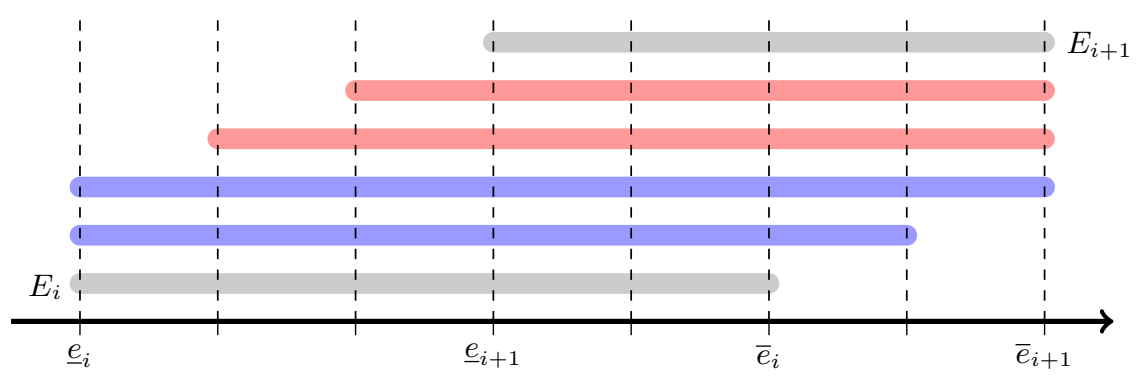

Figure 6 . Construction of $\mathcal{F}_{i}^{-}$(blue sets) and $\mathcal{F}_{i}^{+}$(red sets) from $E_{i}$ and $E_{i+1}$ (in gray).

for any $i=1, \ldots, k$, and let us define the following sets:

$$
\begin{aligned}
& \mathcal{F}_{1}=\left\{\left[x_{1}, x\right] \mid x \in\left[x_{2}, \bar{e}_{1}\right)\right\}, \\
& \mathcal{F}_{k}=\left\{\left[x, x_{n}\right] \mid x \in\left(\bar{e}_{k}, x_{n}\right]\right\}, \\
& \mathcal{F}_{i}^{-}=\left\{\left[\underline{e}_{i}, x\right] \mid x \in\left(\bar{e}_{i}, \bar{e}_{i+1}\right]\right\}, \\
& \mathcal{F}_{i}^{+}=\left\{\left[y, \bar{e}_{i+1}\right] \mid y \in\left(\underline{e}_{i}, \underline{e}_{i+1}\right\},\right.
\end{aligned}
$$

for any $i=2, \ldots, k-1$. The construction of the sets $\mathcal{F}_{i}^{-}$and $\mathcal{F}_{i}^{+}$is pictured in Figure 6.

These sets satisfy the following properties:

(1) $E \prec E_{1}$ for any $E \in \mathcal{F}_{1}$.

(2) $E_{k} \prec E$ for any $E \in \mathcal{F}_{k}$.

(3) $E_{i} \prec E \prec E^{*} \prec E_{i+1}$ for any $E \in \mathcal{F}_{i}^{-}, E^{*} \in \mathcal{F}_{i}^{+}$for $i=2, \ldots, n-1$.

Then, all the elements in $\left\{E_{1}, \ldots, E_{k}\right\} \cup \mathcal{F}_{1} \cup \mathcal{F}_{k} \cup_{i=2}^{k-1}\left(\mathcal{F}_{i}^{-} \cup \mathcal{F}_{i}^{+}\right)$are ordered with respect to $\prec$ and define a p-box $\left(\underline{F}_{2}, \bar{F}_{2}\right)$. According to Proposition 15 , it holds that:

a) If $\mathcal{F}_{1} \cup \mathcal{F}_{k} \bigcup_{i=2}^{k-1}\left(\mathcal{F}_{i}^{-} \cup \mathcal{F}_{i}^{+}\right) \subseteq\left\{E_{1}, \ldots, E_{k}\right\}$, then $\left(\underline{F}_{1}, \bar{F}_{1}\right)=\left(\underline{F}_{2}, \bar{F}_{2}\right)$, and therefore they have the same number of extreme points.

b) Otherwise, $\left(\underline{F}_{2}, \bar{F}_{2}\right)$ has more extreme points than $\left(\underline{F}_{1}, \bar{F}_{1}\right)$.

Let $G_{1} \prec \ldots \prec G_{s}$ denote the focal sets of $\left(\underline{F}_{2}, \bar{F}_{2}\right)$, and let $\mathcal{G}$ be the set of these focal sets. Now, there are two cases:

(1) If $\max \left\{\left|G_{1}\right|, \ldots,\left|G_{s}\right|\right\} \leq 4,\left(\underline{F}_{2}, \bar{F}_{2}\right)$ is a p-box of the Pell family.

(2) If $\max \left\{\left|G_{1}\right|, \ldots,\left|G_{s}\right|\right\}=l>4$, denote by $i_{1}<\ldots<i_{r}$ the indexes such that $\left|G_{i_{1}}\right|=\ldots=\left|G_{i_{s}}\right|=l$. Let us use again the following notation:

$$
\underline{g}_{i_{j}}=\min G_{i_{j}} \text { and } \bar{g}_{i_{j}}=\max G_{i_{j}}
$$

for $j=1, \ldots, r$. Then, by construction, $\left[\underline{g}_{i_{j}-1}, \bar{g}_{i_{j}}\right],\left[\underline{g}_{i_{j}}, \bar{g}_{i_{j}+1}\right] \in \mathcal{F}$ for any $j=1, \ldots, r$. Then, for any $j=1, \ldots, r$, we define the following sets:

$$
G_{i_{j}}^{*}=\left[\underline{g}_{i_{j}+1}, \bar{g}_{i_{j}-1}\right] \text {. }
$$

Then, the sets in $\mathcal{F}^{*}=\mathcal{F} \backslash\left(\left\{G_{i_{1}}, \ldots, G_{i_{r}}\right\}\right) \cup\left\{G_{i_{1}}^{*}, \ldots, G_{i_{r}}^{*}\right\}$ define a p-box $\left(\underline{F}_{3}, \bar{F}_{3}\right)$ which, according to Proposition 12 has more extreme points than $\left(\underline{F}_{2}, \bar{F}_{2}\right)$ and $\max _{G \in \mathcal{F}^{*}}|G|=l^{*}<l$. If $l^{*} \leq 4$, we are in case 1 and get a Pell p-box; otherwise, we repeat the process until we obtain a p-box $\left(\underline{F}^{*}, \bar{F}^{*}\right)$ such that the length of its focal sets is less than or equal to 4 .

In both cases, at the end we obtain a p-box of the Pell family that, as we already know from Theorem 17 , has $\mathcal{P}_{n}$ extreme points. Furthermore, by construction, the only p-boxes with $\mathcal{P}_{n}$ extreme points are those p-boxes which admit no more focal 

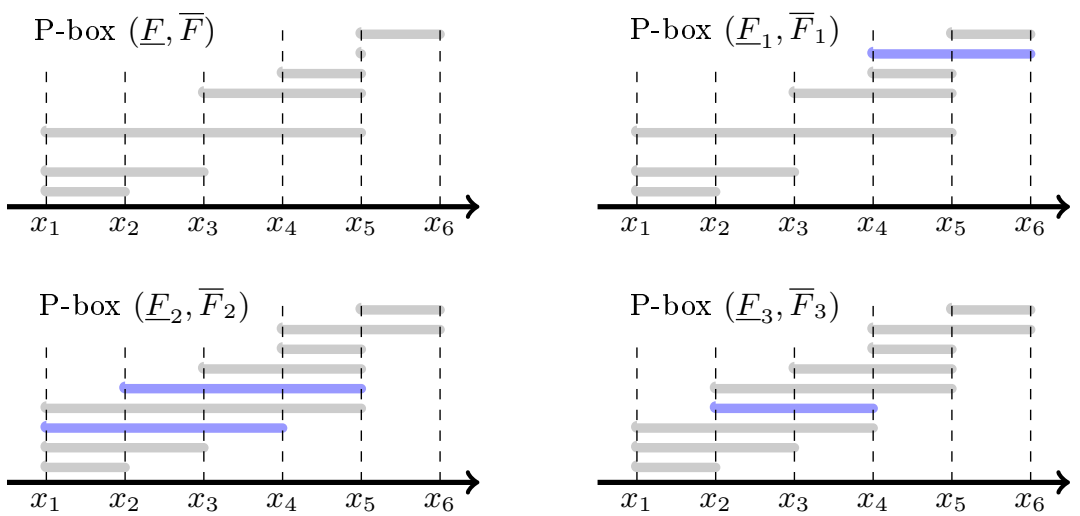

Figure 7. The original p-box $(\underline{F}, \bar{F})$ from Example 19 is depicted in the top-left. Applying Corollary 14 we obtain p-box $\left(\underline{F}_{1}, \bar{F}_{1}\right)$, and using Corollary 15 we obtain the p-box $\left(\underline{F}_{2}, \bar{F}_{2}\right)$. Finally, using Proposition 12 we obtain the p-box $\left(\underline{F}_{3}, \bar{F}_{3}\right)$, that is a p-box from the Pell family. We have drawn in blue the focal sets that are modified or added.

sets and whose length is less than or equal to 4, that is, p-boxes from the Pell family.

As we can see, the number of extreme points of a p-box is strictly lower than $n$ !, but it is at least $2^{n-1}$, as we had already seen in Corollary 11.

Example 19. Consider a p-box $(\underline{F}, \bar{F})$ on $\left\{x_{1}, x_{2}, x_{3}, x_{4}, x_{5}, x_{6}\right\}$ with focal sets:

$$
\begin{gathered}
E_{1}=\left[x_{1}, x_{2}\right], \quad E_{2}=\left[x_{1}, x_{2}, x_{3}\right], \quad E_{3}=\left[x_{1}, x_{5}\right], \quad E_{4}=\left[x_{3}, x_{5}\right], \\
E_{5}=\left[x_{4}, x_{5}\right], \quad E_{6}=\left\{x_{5}\right\}, \quad E_{7}=\left[x_{5}, x_{6}\right] .
\end{gathered}
$$

It can be proven that this p-box has 38 extreme points. Figure 7 illustrates the processes of the previous proof to obtain new p-boxes with a strictly higher number of extreme points. It can be seen that applying Corollaries 14, as well as Propositions 12 and 15 we obtain a p-box from the Pell family that has more extreme points than $(\underline{F}, \bar{F})$.

\section{Counting the number of extreme points of A P-BoX}

Now that we have characterized those p-boxes reaching the maximal number of extreme points a p-box may induce, as well as this maximal number, one problem that remains is whether we can find an Algorithm that exploits the particular structure of p-boxes to find and enumerate those extreme points.

This is what we propose in this section, where we give an Algorithm to grow a tree of depth $n$ whose leaves correspond to the distinct elements of $\mathcal{V}(\mathrm{Bel})$ when Bel is induced by a p-box.

In this section, we provide an algorithm to enumerate the extreme points of a given p-box. This algorithm that builds a tree consists in incrementally forming vectors $\vec{v} \in \mathcal{V}(\mathrm{Bel})$ by successively assigning values $v_{i}$ to their elements. The $i$ th level of the tree corresponds to $v_{i}$ values, and each leaf then corresponds to a distinct extreme point (whose values can be found back by going from the leaf to the root). Pseudo-Algorithm 3 describes how children are created from a node having depth $d<n$. The idea of the algorithm is, for a given depth $d$, to make 
as many branches as the value $v_{d+1}$ that vectors $v \in \mathcal{V}(\mathrm{Bel})$ can take, knowing the values $v_{1}, \ldots, v_{d}$ defined by the parents of this node in the tree. At a given depth $d$, a node is created (Loop 4-22 of Algorithm 3) for each possible number of focal elements that can assign their masses to $x_{d+1}$ (including 0 ), and the created node receives the corresponding probability $P\left(\left\{x_{d+1}\right\}\right)$ as well as the value $v_{d+1}$ of the corresponding permutation vector in $\mathcal{V}$, and the update set of focal elements determining which mass remains to be distributed to which possible elements. Note that since all focal sets of $\mathrm{Bel}$ are distinct, the value $\ell^{*}$ (line 7 ) will be different for all numbers in $\underline{N b}, \ldots, \overline{N b}$ that $\vec{v}_{d+1}$ can take. This ensures that every such number correspond to a possible permutation (hence to an extreme point) where $x_{d+1} \leq_{\sigma} x_{j}$ for $j \in\left\{d+2, \ldots, \ell^{*}\right\}$, hence to an extreme point of the p-box. The whole tree can then be built by applying this method recursively, until a depth $n$ is reached. The root node (level 0 ) simply starts with $\mathcal{E}=\mathcal{F}$.

The idea of the algorithm is as follows: assume that for $x_{1}$, the only focal sets in $\mathcal{F}$ containing it are $E_{1}=\left\{x_{1}\right\}, E_{2}\left\{x_{1}, x_{2}\right\}$ and $E_{3}=\left\{x_{1}, x_{2}, x_{3}, x_{4}\right\}$. If $v_{1}=2$, then it necessarily means that $m\left(E_{1}\right)$ and $m\left(E_{2}\right)$ are assigned to $x_{1}$, and $m\left(E_{3}\right)$ to another element, concluding that $P\left(\left\{x_{1}\right\}\right)=m\left(E_{1}\right)+m\left(E_{2}\right)$ for this path. This is formalized by Lines 5-6 in Algorithm 3. $v_{1}=2$ also necessary means that $x_{2}$ is, in the corresponding permutation, after $x_{1}$, otherwise $E_{2}$ would not have been assigned to $x_{1}$, and that $x_{3}$ or $x_{4}$ is before $x_{1}$ in the permutation, so we can safely remove $x_{2}$ and $x_{1}$ from all sets containing $x_{3}, x_{4}$ in addition to removing $E_{1}, E_{2}$ whose mass have been affected, which is equivalent to all the sets containing $x_{2}$ after $E_{3}$. When $v_{1}=3$, then all we know is that $x_{2}, x_{3}, x_{4}$ are after $x_{1}$ in the permutation, and we can only remove $E_{1}, E_{2}, E_{3}$, without changing the other sets (as we do not know the ordering between $x_{2}, x_{3}, x_{4}$ ). This update corresponds to Lines 9 to 21 in Algorithm 3. Example 20 provides a more complete illustration of the mechanism.

Example 20. Consider a p-box $(\underline{F}, \bar{F})$ on $\left\{x_{1}, x_{2}, x_{3}, x_{4}\right\}$ whose focal sets are given by:

$$
\begin{aligned}
& E_{1}=\left\{x_{1}\right\}, \quad m\left(E_{1}\right)=0.2 . \\
& E_{2}=\left\{x_{1}, x_{2}, x_{3}\right\}, \quad m\left(E_{2}\right)=0.1 . \\
& E_{3}=\left\{x_{1}, x_{2}, x_{3}, x_{4}\right\}, \quad m\left(E_{3}\right)=0.4 . \\
& E_{4}=\left\{x_{3}, x_{4}\right\}, \quad m\left(E_{4}\right)=0.3 .
\end{aligned}
$$

Following the previous steps, the number of focal sets that can be assigned to $x_{1}$ lies between 1 and 3 .

$v_{1}=3$ : In this case, $P\left(\left\{x_{1}\right\}\right)=0.7$. Updating the focal sets using Line 15-17, we only obtain one updated focal set $E_{4}=\left\{x_{3}, x_{4}\right\}$ such that $m\left(E_{4}\right)=0.3$, having removed $E_{1}, E_{2}, E_{3}$. We necessarily have along this path $v_{2}=0$ (only one children is created), and $v_{3}$ can either be 0 or 1 . Then, we obtain two extreme points, given by:

$$
P_{1}=(0.7,0,0.3,0), \quad P_{2}=(0.7,0,0,0.3) \text {. }
$$

and corresponding respectively to $v_{3}=1, v_{3}=0$

$v_{1}=2$ : In this case, $P\left(\left\{x_{1}\right\}\right)=0.3$. If $v_{1}=2$, this means that $x_{4}$ appears before $x_{1}$ in the permutation assigned to the vector $v$, but that $x_{2}$ and $x_{3}$ appear after it. Then, after updating the focal sets, we only obtain $E_{3}=\left\{x_{4}\right\}$ and $E_{4}=\left\{x_{4}\right\}$, that we merge into $E_{4}=\left\{x_{4}\right\}$ with mass $m\left(E_{4}\right)=0.7$. Then, we obtain one extreme point:

$$
P_{3}=(0.3,0,0,0.7)
$$




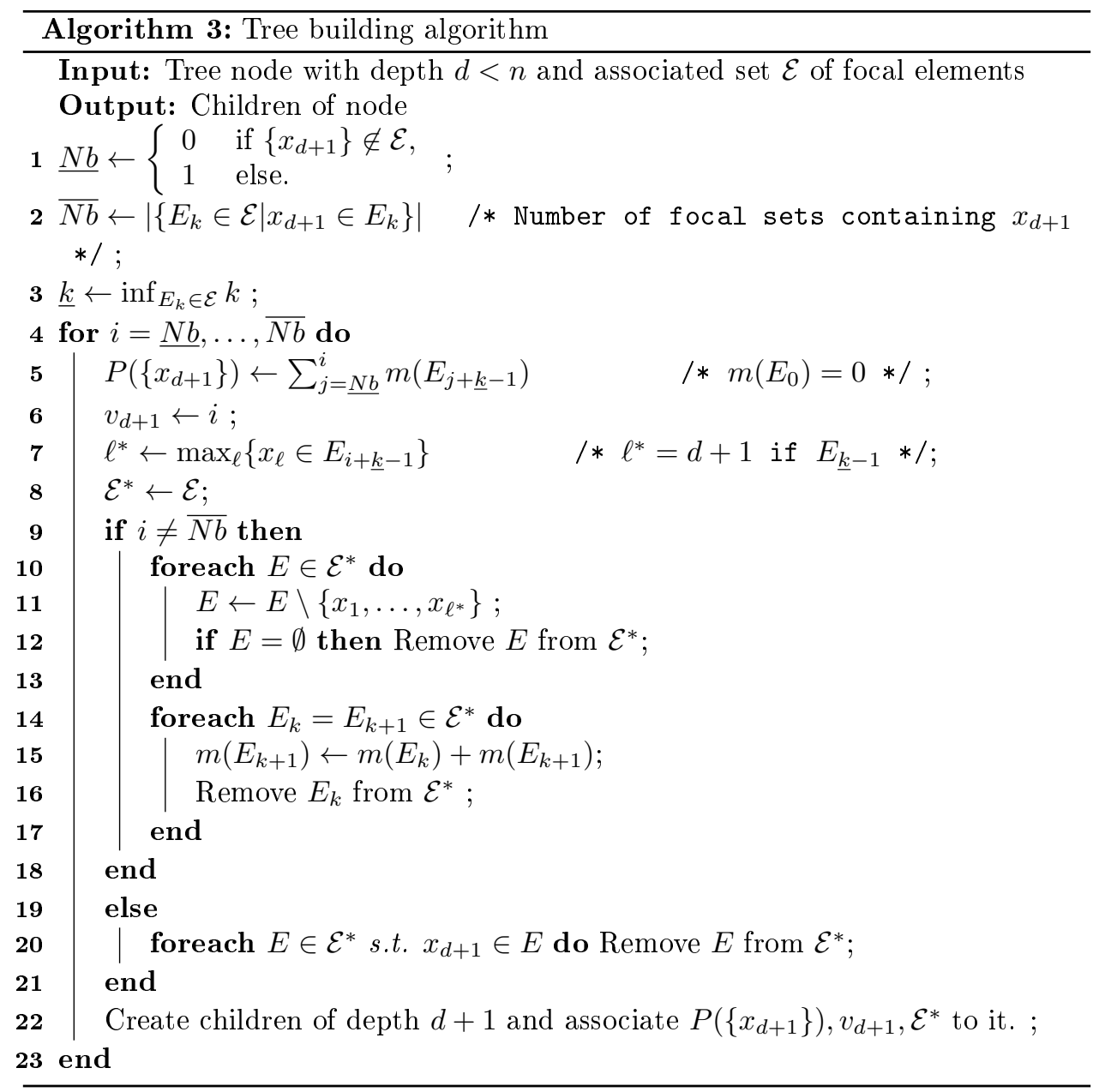

$v_{1}=1$ : In this case, $P\left(\left\{x_{1}\right\}\right)=0.2$. Updating the focal sets, we obtain:

$$
\begin{aligned}
& E_{2}=\left\{x_{2}, x_{3}\right\}, \quad m\left(E_{2}\right)=0.1 . \\
& E_{3}=\left\{x_{2}, x_{3}, x_{4}\right\}, \quad m\left(E_{3}\right)=0.4 . \\
& E_{4}=\left\{x_{3}, x_{4}\right\}, \quad m\left(E_{4}\right)=0.3 .
\end{aligned}
$$

Now, we apply again Algorithm 3 with these three focal sets. For this aim, note that $N b=0$ and $\overline{N b}=2$ :

$v_{2}=2$ : In this case $P\left(\left\{x_{2}\right\}\right)=0.5$ and updating the focal sets we only obtain $E_{4}=\left\{x_{3}, x_{4}\right\}$ with mass 0.3 . Similarly to the case $v_{1}=3$, we obtain two extreme points:

$$
P_{4}=(0.2,0.5,0.3,0), \quad P_{5}=(0.2,0.5,0,0.3) .
$$

$v_{2}=1$ : In this case $P\left(\left\{x_{2}\right\}\right)=0.1$ and updating the focal sets we only obtain (after merging of identical sets) $E_{4}=\left\{x_{4}\right\}$ with mass 0.7 . Then we obtain one extreme point:

$$
P_{6}=(0.2,0.1,0,0.7) \text {. }
$$

$v_{2}=0$ : In this case $P\left(\left\{x_{2}\right\}\right)=0$. If we update the focal sets, we obtain:

$$
\begin{aligned}
& E_{3}=\left\{x_{3}\right\}, \quad m\left(E_{3}\right)=0.1 . \\
& E_{4}=\left\{x_{3}, x_{4}\right\}, \quad m\left(E_{4}\right)=0.7 .
\end{aligned}
$$




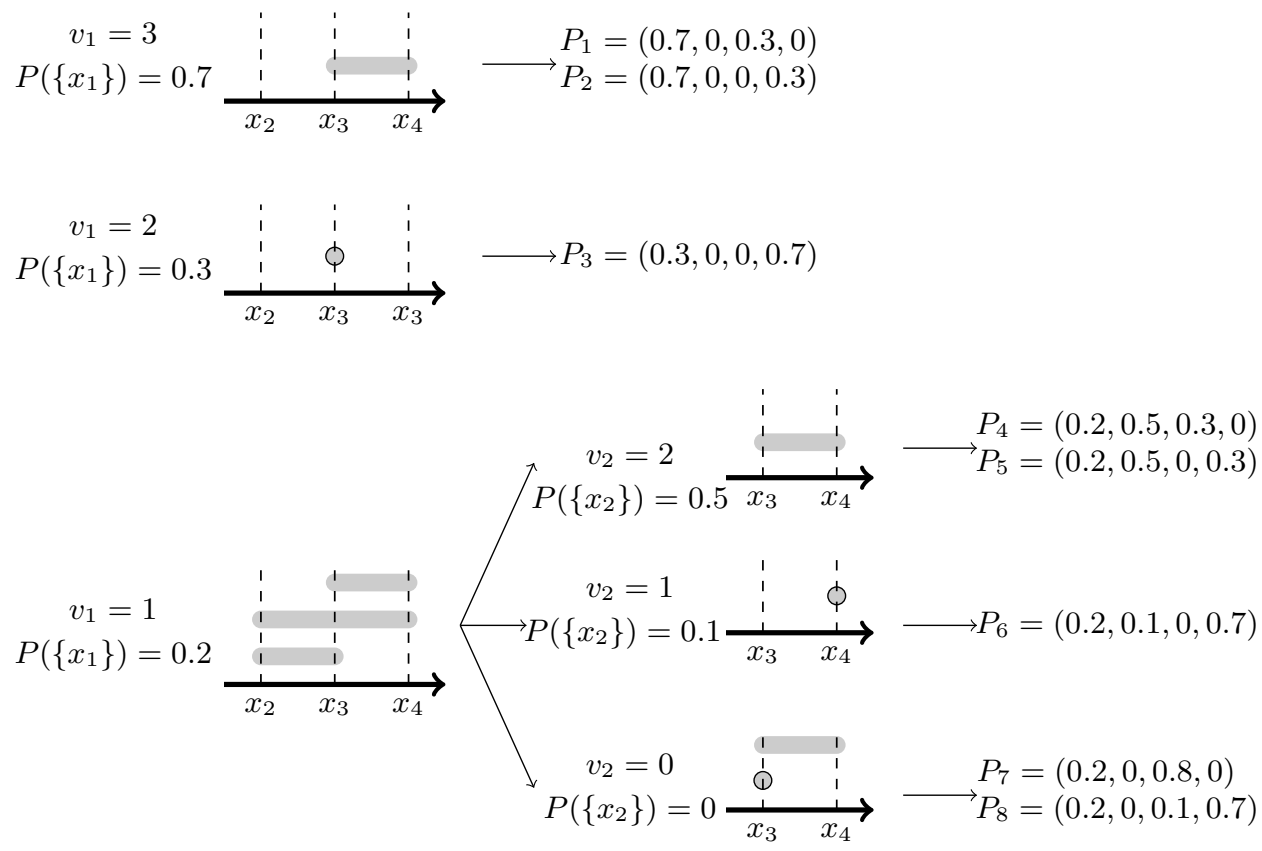

FiguRE 8. Algorithm for extracting the extreme points.

Applying again Algorithm 3 to these sets, we obtain the following extreme points:

$$
P_{7}=(0.2,0,0.8,0), \quad P_{8}=(0.2,0,0.1,0.7) .
$$

We have depicted this procedure in Figure 8, developing only those branches that are non-trivial, that is the first level of the tree and the second level when $v_{1}=1$.

Finally, let us note that the algorithm will have one leaf per extreme point, meaning that its complexity is linear in the number $m$ of such extreme points. In the worst case, this number is the Pell number, which grows exponentially with $n$, rather than super-exponentially (as the general maximum number of extreme points is $n$ !). Also, we can expect that, in general, practical p-boxes will have a quite lower number of extreme points.

\section{Conclusions}

This paper mainly deals with the problem of characterizing, and finding, the extreme points of p-boxes. The main theoretical result of the paper is that the maximal number of extreme points of a p-box defined on $\left\{x_{1}, \ldots, x_{n}\right\}$ is the Pell number $\mathcal{P}_{n}$, and that only those specific p-boxes of the Pell family have this number of extreme points. To do so, we have also provided a number of what we believe to be new and interesting results about the number of extreme points of belief functions, among which a new characterization in terms of "counting" vectors and a specification of the condition under which a belief function has $n$ ! extreme points. These counting vectors also show that the number of extreme points of a belief function only depends on the focal sets, but they are irrelevant of their associated mass.

On the more practical side, we have provided a tree-growing algorithm allowing one to enumerate and find the extreme points associated to a p-box. It should 
be noted that our results somehow complement those obtained in the continuous case [28], and allow one to work, for example, in a discretized setting. Possible extensions of this work include the study of extreme points of bivariate p-boxes [22], which unfortunately are not special cases of belief functions in general (yet, first studies could focus on those cases where bivariate p-boxes are also belief functions, such as comonotone $[16,17]$ or maxitive bivariate p-boxes [19]).

\section{ACKNOWLEDGEMENTS}

The research reported in this paper has been supported by project TIN201459543-P, and by the project Labex MS2T, financed by the French Government through the program "Investments for the future" managed by the National Agency for Research (Reference ANR-11-IDEX-0004-02). We also want to thank Georg Schollmeyer for stimulating discussion on the topic of this paper and the reviewers for their helpful comments.

\section{REFERENCES}

[1] A. M Aboalkhair, F. P. A. Coolen, and I. M. MacPhee. Nonparametric predictive reliability of series of voting systems. European Journal of Operational Research, 226(1):77-84, 2013.

[2] S. Angilella, S. Greco, and B. Matarazzo. Non-additive robust ordinal regression: A multiple criteria decision model based on the Choquet integral. European Journal of Operational Research, 201(1):277-288, 2010.

[3] A. Chateauneuf and J.-Y. Jaffray. Some characterizations of lower probabilities and other monotone capacities through the use of Möbius inversion. Mathematical Social Sciences, 17(3):263-283, 1989.

[4] F. P. A. Coolen and L. V. Utkin. Reliability and risk, pages 305-317. John Wiley \& Sons, Ltd, 2014.

[5] F. G Cozman. Credal networks. Artificial intelligence, 120(2):199-233, 2000.

[6] Fabio G Cozman and Peter Walley. Graphoid properties of epistemic irrelevance and independence. Annals of Mathematics and Artificial Intelligence, 45(1):173-195, 2005.

[7] L. M. de Campos, J. F. Huete, and S. Moral. Probability intervals: a tool for uncertain reasoning. International Journal of Uncertainty, Fuziness and Knowledge-Based Systems, 2:167-196, 1994.

[8] A. P. Dempster. Upper and lower probabilities induced by a multivalued mapping. Annals of Mathematical Statistics, 38:325-339, 1967.

[9] S. Destercke and D. Dubois. Special cases, pages 79-92. John Wiley \& Sons, Ltd, 2014.

[10] S. Destercke, D. Dubois, and E. Chojnacki. Unifying practical uncertainty representations: I. Generalized p-boxes. International Journal of Approximate Reasoning, 49(3):649-663, 2008.

[11] S. Destercke and G. Yang. Cautious ordinal classification by binary decomposition. In $M a-$ chine Learning and Knowledge Discovery in Databases, pages 323-337. Springer, 2014.

[12] S. Ferson, V. Kreinovich, L. Ginzburg, D. S. Myers, and K. Sentz. Constructing probability boxes and Dempster-Shafer structures. Technical Report SAND2002-4015, Sandia National Laboratories, January 2003.

[13] E. Kriegler. Utilizing belief functions for the estimation of future climate change. International Journal of Approximate Reasoning, 39(2-3):185-209, 2005.

[14] E. Miranda, I. Couso, and P. Gil. Extreme points of credal sets generated by 2-alternating capacities. International Journal of Approximate Reasoning, 33(1):95-115, 2003.

[15] P. Miranda, E. F. Combarro, and P. Gil. Extreme points of some families of non-additive measures. European journal of operational research, 174(3):1865-1884, 2006.

[16] I. Montes and S. Destercke. Comonotone lower probabilities for bivariate and discrete structures. In Proceedings of the Ninth International Symposium on Imprecise Probability: Theories and Applications, 2015.

[17] I. Montes and S. Destercke. Comonotonicity for sets of probabilities. Fuzzy Sets and Systems, 2016.

[18] I. Montes and S. Destercke. On extreme points of p-boxes and belief functions. In Proceedings of SMPS Conference, 2016.

[19] I. Montes and E. Miranda. Bivariate p-boxes and maxitive functions. In International Conference on Information Processing and Management of Uncertainty in Knowledge-Based Systems, pages 141-152. Springer, 2016. 
[20] I. Montes, E. Miranda, and S. Montes. Decision making with imprecise probabilities and utilities by means of statistical preference and stochastic dominance. European Journal of Operational Research, 234(1):209-220, 2014.

[21] Serafín Moral and Andrés Cano. Strong conditional independence for credal sets. Annals of Mathematics and Artificial Intelligence, 35(1-4):295-321, 2002.

[22] R. Pelessoni, P. Vicig, I. Montes, and E. Miranda. Bivariate p-boxes. International Journal of Uncertainty, Fuzziness and Knowledge-Based Systems, 24(02):229-263, 2016.

[23] G. Schollmeyer. On the number and characterization of the extreme points of the core of necessity measures on finite spaces. In T. Augustin, S. Doria, E. Miranda, and E. Quaeghebeur, editors, ISIPTA '15 - Proceedings of the Ninth International Symposium on Imprecise Probability: Theories and Applications. SIPTA, 2015.

[24] G. Shafer. A Mathematical Theory of Evidence. Princeton University Press, Princeton, NJ, 1976.

[25] Christophe Simon and Frédérique Bicking. Hybrid computation of uncertainty in reliability analysis with p-box and evidential networks. Reliability Engineering \& System Safety, 2017.

[26] M. C. M. Troffaes and S. Destercke. Probability boxes on totally preordered spaces for multivariate modelling. International Journal of Approximate Reasoning, 52(6):767-791, 2011.

[27] M. C. M. Troffaes, E. Miranda, and S. Destercke. On the connection between probability boxes and possibility measures. Information Sciences, 224:88-108, 2013.

[28] L. Utkin and S. Destercke. Computing expectations with continuous p-boxes: Univariate case. International Journal of Approximate Reasoning, 50(5):778-798, 2009.

[29] P. Walley. Statistical Reasoning with Imprecise Probabilities. Chapman and Hall, London, 1991.

[30] G. Walter and T. Augustin. Imprecision and prior-data conflict in generalized bayesian inference. Journal of Statistical Theory and Practice, 3(1):255-271, 2009. 